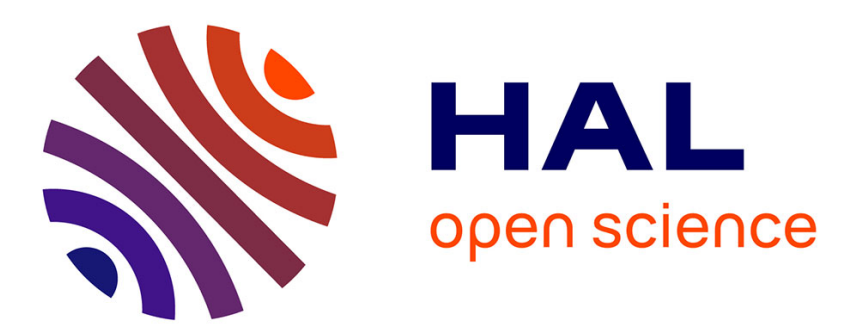

\title{
The Resource-Constrained Activity Insertion Problem with Minimum and Maximum Time Lags
}

Christian Artigues, Cyril Briand

\section{To cite this version:}

Christian Artigues, Cyril Briand. The Resource-Constrained Activity Insertion Problem with Minimum and Maximum Time Lags. Journal of Scheduling, 2009, 12, pp.447-460. 10.1007/s10951-0090124-x . hal-00194384v2

\section{HAL Id: hal-00194384 \\ https://hal.science/hal-00194384v2}

Submitted on 1 Sep 2008

HAL is a multi-disciplinary open access archive for the deposit and dissemination of scientific research documents, whether they are published or not. The documents may come from teaching and research institutions in France or abroad, or from public or private research centers.
L'archive ouverte pluridisciplinaire HAL, est destinée au dépôt et à la diffusion de documents scientifiques de niveau recherche, publiés ou non, émanant des établissements d'enseignement et de recherche français ou étrangers, des laboratoires publics ou privés. 


\title{
The Resource-Constrained Activity Insertion Problem with Minimum and Maximum Time Lags
}

\author{
Christian Artigues • Cyril Briand
}

Received: date / Accepted: date

\begin{abstract}
This paper considers the resource-constrained activity insertion problem with minimum and maximum time lags. The problem consists in inserting a single activity in a partial schedule while preserving its structure represented through resource flow networks and minimizing the makespan increase caused by the insertion. In the general case, we show that finding a feasible insertion that minimizes the project duration is NP-hard. When only minimum time lags are considered and when activity durations are strictly positive, we show the problem is polynomially solvable, generalizing previously established results on activity insertion for the standard resourceconstrained project scheduling problem.
\end{abstract}

Keywords Resource-constrained project scheduling · minimum and maximum time lags $\cdot$ activity insertion problem $\cdot$ complexity

\section{Introduction}

In the standard resource-constrained project scheduling problem (RCPSP), the precedence relations are simple: an activity cannot start before the end of all its predecessors. In other words, between the start time of an activity $A_{i}$ and the start time of its successor $A_{j}$ there is a minimal distance (or minimum time lag) equal to the duration of $A_{i}$. The RCPSP with minimum and maximum time lags (RCPSP/max) involves generalized precedence relations where the minimum time lag between $A_{i}$ and $A_{j}$ can be any non-negative value and where there is possibly a maximum allowed time lag between the start time of $A_{i}$ and the start time of $A_{j}$.

Independently of makespan minimization, deciding whether it exists a resourcefeasible schedule that respects both the minimum and maximum time lags is NPcomplete [3] whereas, for the standard RCPSP, this problem is polynomial. For that reason, the RCPSP/max problem has been extensively studied in the scheduling literature. For a thorough analysis of this problem, we recommend the book [12]. In this paper, unlike most approaches that focused on finding a global schedule that minimizes

C. Artigues - C. Briand

LAAS-CNRS, Université de Toulouse, 7 avenue du Colonel Roche, 31077 Toulouse, France

E-mail: artigues,briand@laas.fr 
the project duration, we illustrate the difficulties brought by the maximum time lags by considering the apparently simple problem of inserting a single activity inside an existing schedule.

This problem aims at finding an insertion for this activity satisfying the minimum/maximum time lags and resource constraints, while preserving the partial schedule structure. The objective is to minimize the project duration increase. Such a problem arises in local search procedures for reinsertion neighborhoods that unschedule an activity and insert it at another position [2,4,13]. For such procedures finding a compromise between the quality of the reinsertion operator and its computational requirements is a fundamental issue. Since the last decades, there has been a growing interest in the predictive-reactive scheduling framework to take account of uncertainty in scheduling. We refer to recent surveys in the context of manufacturing systems [14] and project management [15]. Inserting efficiently unexpected activities in an existing schedule is among the central issues raised by predictive-reactive scheduling reactive $[2,1,5,8]$.

The interest of preserving the structure of the partial schedule is twofold. First, the size of the search space is decreased, which is useful both for neighborhood search and reactive scheduling. Second, this policy tends to minimize the disturbances during the on-line project execution phase, which is referred to as ensuring the schedule stability.

In $[4,13]$ among others, operation insertion problems are solved in a generalized job-shop. In [9], the job insertion problem in job-shop scheduling is studied. In [8] and [10], insertion problems in a general disjunctive scheduling framework capturing a variety of job shop scheduling problems and insertion types are considered. A polyhedral characterization of feasible insertions is provided and lower and upper bounding procedures are developed. Since disjunctive and job-shop scheduling are particular cases of the RCPSP where all resources have unit availability, the proposed procedures cannot be used to solve the problem considered in this paper.

For the RCPSP and the RCPSP/max, we can define the structure of the schedule by means of resource flow networks, which induce additional precedence constraints preventing resource over-subscription. With such a structure, the activity insertion problem has been addressed for the standard RCPSP in [1,2]. An $\mathrm{O}\left(n^{2} m\right)$ optimal insertion procedure has been proposed where $n$ is the number of activities and $m$ is the number of resources. In [1], a tabu search procedure based on the reinsertion of a critical activity in the current resource flow network has been designed and obtained good results to solve the standard RCPSP. Hence, an important issue is to establish whether this approach can be extended to minimum and maximum time lags for proposing a new class of neighborhood search method for the RCPSP/max.

In this paper, we show that the considered insertion problem is NP-hard in the general case. However, when maximum time lags are ignored, a new polynomial algorithm is issued, generalizing the results obtained in $[1,2]$.

The resource-constrained activity insertion problem is presented in Section 2. The concept of feasible insertion is introduced in Section 3. Feasibility conditions are described in Section 4. The problem complexity is given in Section 5. The particular case where only minimum time lags are considered is studied in Section 6. Concluding remarks are drawn in Section 7. 


\section{Problem statement}

Activities constituting the project are identified by set $V=\left\{A_{0}, \ldots, A_{n+1}\right\}$. Activity $A_{0}$ represents by convention the start of the schedule and activity $A_{n+1}$ represents symmetrically the end of the schedule. The set of non-dummy activities is identified by $\mathcal{A}=\left\{A_{1}, \ldots, A_{n}\right\} \cdot p_{i}$ denotes the duration of activity $i$ with $p_{0}=p_{n+1}=0$. We assume in this paper that $p_{i}>0, \forall A_{i} \in \mathcal{A}$.

A valuated activity-on-node graph $G(V, E, l)$ is defined where nodes correspond to activities and arcs correspond to precedence relations. Each arc $\left(A_{i}, A_{j}\right) \in E$ is valuated by an integer time lag $l_{i j}$. The case $l_{i j} \geq 0$ corresponds to a minimum time lag of $l_{i j}$ units stating that $A_{j}$ has to start at least $l_{i j}$ time units after the start time of $A_{i}$. In the standard RCPSP, only minimum time lags are considered and $l_{i j}=p_{i}$ for each arc $\left(A_{i}, A_{j}\right) \in E$. The case $l_{i j} \leq 0$ corresponds to a maximum time lag units stating that $A_{i}$ has to start at the latest $-l_{i j}$ time units after the start time of $A_{j}$. Resource constraints are defined as in the standard RCPSP: $\mathcal{R}=\left\{R_{1}, \ldots, R_{m}\right\}$ denotes the set of $m$ resources. $B_{k}$ denotes the availability of $R_{k} . b_{i, k}$ represents the amount of resource $R_{k}$ used during the execution of $A_{i}$.

A solution is a non-preemptive schedule giving the start time $S_{i}$ of each activity $A_{i}$. It is assumed that $G$ includes a path of length at least 0 between $A_{0}$ and each activity $A_{i}$ and a path of length at least $p_{i}$ between each activity $A_{i}$ and $A_{n+1}$.

The RCPSP with minimum and maximum time lags is the problem $(P)$ of finding a non-preemptive schedule of minimal makespan $S_{n+1}$ subject to precedence and resource constraints.

$$
\begin{gathered}
\min S_{n+1} \\
S_{j}-S_{i} \geq l_{i j} \quad \forall\left(A_{i}, A_{j}\right) \in E \\
\sum_{A_{i} \in \mathcal{A}_{t}} b_{i, k} \leq B_{k} \quad \forall R_{k} \in \mathcal{R}, \forall t \geq 0
\end{gathered}
$$

A schedule $S$ is feasible if it satisfies the generalized precedence constraints 2 and the resource constraints 3 where $\mathcal{A}_{t}=\left\{i \in \mathcal{A} \mid S_{i} \leq t<S_{i}+p_{i}\right\}$ represents the set of non-dummy activities in process at time $t$.

$(P)$ does not necessarily have a solution and, unfortunately, deciding whether $(P)$ has a solution is NP-complete [3].

Infeasibility can be due to an inconsistency of the precedence constraints $E$, independently of the resource constraints. More precisely, there is no solution to $(P)$ if there is a cycle of positive length in $G(V, E, l)$. The Floyd-Warshall (FW) algorithm can be used to detect in $\mathcal{O}\left(n^{3}\right)$ time such a cycle. In the remaining of the paper, only problems for which the temporal constraints are consistent are considered.

In case there is no cycle of positive length in $G(V, E, l)$, a distance matrix $\left(\delta_{i, j}\right)_{i, j \in V^{2}}$ can be computed where $\delta_{i, j}$ is the length of the longest path from $A_{i}$ to $A_{j}$ in $G(V, E, l)$. $\left(\delta_{i, j}\right)$ values are returned by $\mathrm{FW}$. By convention, $\Delta_{i, j}=-\infty$ means that there is no path from $i$ to $j$.

We use the concept of resource-flow network to represent the solutions to $(P)[1,2$, $6,11,12]$. A resource flow $f$ is a $(n+2) \times(n+2) \times m$ matrix verifying equations $(4-6)$ defined below:

$$
f_{i, j, k} \geq 0 \quad \forall A_{i} \in \mathcal{A} \cup\left\{A_{0}\right\}, \forall A_{j} \in \mathcal{A} \cup\left\{A_{n+1}\right\}, \forall R_{k} \in \mathcal{R}
$$




$$
\begin{gathered}
\sum_{A_{i} \in \mathcal{A} \cup\left\{A_{n+1}\right\}} f_{0, i, k}=\sum_{A_{i} \in \mathcal{A} \cup\left\{A_{0}\right\}} f_{i, n+1, k}=B_{k} \quad \forall R_{k} \in \mathcal{R} \\
\sum_{A_{j} \in \mathcal{A} \cup\left\{A_{n+1}\right\}} f_{i, j, k}=\sum_{A_{j} \in \mathcal{A} \cup\left\{A_{0}\right\}} f_{j, i, k}=b_{i, k} \quad \forall A_{i} \in \mathcal{A}, \forall R_{k} \in \mathcal{R}
\end{gathered}
$$

$f_{i, j, k}$ denotes the number of resource $R_{k}$ units transferred from activity $A_{i}$ to activity $A_{j}$.

From flow $f$, a set of precedence constraints (or arcs) $F(f)$ can be defined as

$$
F(f)=\left\{\left(A_{i}, A_{j}\right) \in V^{2} \mid \exists R_{k} \in \mathcal{R}, f_{i, j, k}>0\right\}
$$

$F(f)$ is the set of precedence constraints (or arcs) induced by flow $f$. We say that $A_{j}$ is a resource successor of $A_{i}$ and $A_{i}$ is a resource predecessor of $A_{j}$ if $\left(A_{i}, A_{j}\right) \in F(f)$.

From arc set $F(f)$, we define the graph induced by flow $f$ as

$$
\mathcal{G}(f)=G(V, E \cup F(f), L(f))
$$

where arc valuation $L(f): E \cup F(f) \rightarrow \mathbb{N}$ is defined as:

$$
L_{i j}(f)= \begin{cases}l_{i j} & \text { if }\left(A_{i}, A_{j}\right) \in E,\left(A_{i}, A_{j}\right) \notin F(f) \\ p_{i} & \text { if }\left(A_{i}, A_{j}\right) \in F(f),\left(A_{i}, A_{j}\right) \notin E \\ \max \left(p_{i}, l_{i j}\right) & \text { if }\left(A_{i}, A_{j}\right) \in E \cap F(f)\end{cases}
$$

Now, let $\left(\Delta_{i, j}(f)\right)_{A_{i}, A_{j} \in V}$ denote the distance matrix induced by flow $f . \Delta_{i, j}(f)$ is the length of the longest path from $A_{i}$ to $A_{j}$ in $\mathcal{G}(f)$. The precedence constraints $F(f)$ induced by the flow are consistent if and only if there is no cycle of positive length in $\mathcal{G}(f)$. In particular, $\Delta_{0, i}(f), \forall A_{i} \in V$ denotes the earliest start schedule associated with the flow $f$. The following formulation of the RCPSP/max can be derived from the flow concept (see also Theorems 1 and 2 in [11] and Section 2.13 in [12]).

Definition 1 (RCPSP/max) $(P)$ can be defined as the problem of finding a resource flow $f$ verifying (4-6), such that $\mathcal{G}(f)$ contains no cycle of positive length and such that $\Delta_{0, n+1}(f)$ is minimal.

Hence, a flow $f$ verifying (4-6), such that $\mathcal{G}(f)$ contains no cycle of positive length is said to be feasible. It corresponds to a solution to $(P)$ giving feasible schedule $\left(\Delta_{0, i}(f)\right)_{A_{i} \in V}$.

To define the resource constrained activity insertion problem (RCAIP), consider a partial solution flow $f$ in which all activities but one, denoted by $A_{x}(0<x<n+1)$, have been scheduled. This amounts to considering a complete solution $f$ to problem $\left(\mathrm{P}_{-x}\right)$ identical to $(P)$ except that $b_{x, k}=0, \forall R_{k} \in \mathcal{R}$. The RCAIP amounts to compute a solution to $(P)$ by inserting activity $A_{x}$ in the flow $f$ associated with the solution of $\left(\mathrm{P}_{-x}\right)$ in such a way that the resource flow assigned to $A_{x}$ can only be rerouted from $f$. The concept of rerouted flow can be formally defined as follows:

Definition 2 (Rerouted flow) A flow $f^{\prime}$ is rerouted toward a flow $f$ for an activity $A_{x}$ if there exists values $q_{i, j, k}$, for all $i, j \in V$ and for all $R_{k} \in \mathcal{R}$ such that $0 \leq q_{i, j, k} \leq$ $f_{i, j, k}$ and

$$
\begin{array}{cc}
f_{x, j, k}^{\prime}=\sum_{A_{i} \in V} q_{i, j, k} & \forall A_{j} \in V, \forall R_{k} \in \mathcal{R} \\
f_{i, x, k}^{\prime}=\sum_{A_{j} \in V} q_{i, j, k} & \forall A_{i} \in V, \forall R_{k} \in \mathcal{R} \\
f_{i, j, k}^{\prime}=f_{i, j, k}-q_{i, j, k} & \forall A_{i}, A_{j} \in V, \forall R_{k} \in \mathcal{R}
\end{array}
$$


From this definition, the resource-constrained activity insertion problem $\left(\mathrm{P}_{x}\right)$ can be defined as follows.

Definition 3 (RCAIP) Given a flow $f$, feasible for $\left(P_{-x}\right)$, the resource-constrained activity insertion problem $\left(P_{x}\right)$ amounts to find a rerouted flow $f^{\prime}$ towards $A_{x}$, feasible for $(P)$, such that $\Delta_{0, n+1}\left(f^{\prime}\right)$ is minimized.

We have $\Delta_{0, n+1}\left(f^{\prime}\right) \geq \Delta_{0, n+1}(f)$ since by transitivity, any precedence constraint induced by $f$ is also induced by $f^{\prime}$. Note that an instance of RCAIP is given by tuple $(n, m, p, \delta, b, B, x, f)$.

Consider an example issued from [12], comprising five real activities and a single resource of three units . Durations and resource demands are given in Table 1. Minimum and maximum time lags and the corresponding the project network are displayed on the left side of Figure 1.

Table 1 Activity durations and resource demands

\begin{tabular}{llllllll}
\hline$A_{i}$ & 0 & 1 & 2 & 3 & 4 & 5 & 6 \\
$p_{i}$ & 0 & 6 & 4 & 2 & 4 & 2 & 0 \\
$b_{i}$ & 0 & 1 & 2 & 2 & 2 & 3 & 0 \\
\hline
\end{tabular}
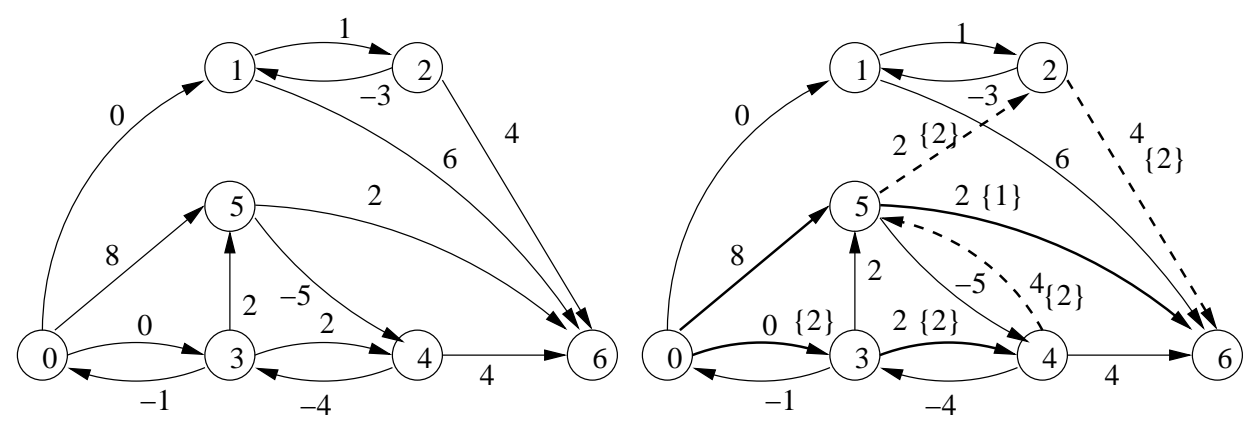

Fig. 1 Minimum/maximum time lags (left side) and flow $f$ solution to $\left(\mathrm{P}_{-1}\right)$ (right side)

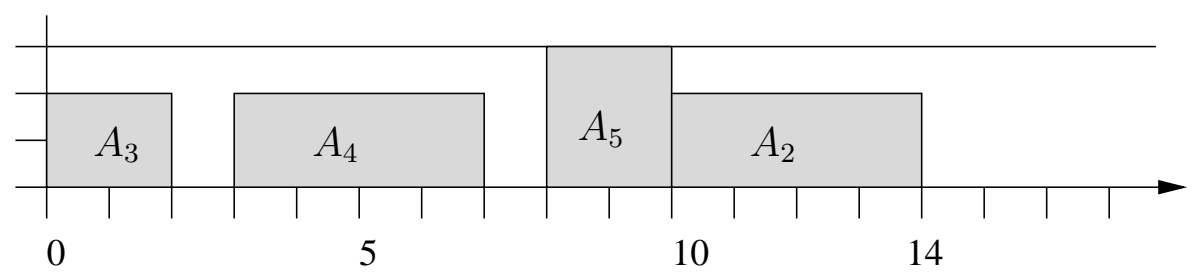

Fig. 2 Gantt chart of the solution to $\left(\mathrm{P}_{-1}\right)$

A partial schedule for $(P)$, in which activity $A_{1}$ is not scheduled, corresponding to a complete solution of $\left(\mathrm{P}_{-1}\right)$, is displayed on Figure 2. Resource flow $f$, corresponding 
to this schedule, is displayed on the right side of Figure 1. Thin arcs correspond to the original precedence constraints only, while thick arcs are induced by the flow (the flow value is displayed between braces). Plain thick arcs belong to $E \cup F(f)$ while thick dotted arcs are only induced by the flow. As displayed on Figure 1, the non-zero flow amounts of $f$ are $f_{0,3}=2, f_{0,5}=1, f_{3,4}=2, f_{4,5}=2, f_{5,6}=1, f_{5,2}=2, f_{2,6}=2$. The activity candidate for insertion $A_{1}$ is such that $b_{i}=1$. Hence, to insert $A_{1}$ into $f$ according to the insertion scheme described above, we have to find activities $A_{i}$ and $A_{j}$ such that the amount of flow taken from $f_{i, j}$ and rerouted to $A_{1}$ is $q_{i, j}=1$. The reader may check that among the non zero flow amounts listed above, the only valid candidates are $A_{i}=A_{5}$ and $A_{j}=A_{6}$. Indeed, inserting $A_{1}$ before activity $A_{5}$ or between $A_{2}$ and $A_{5}$ violates constraint $S_{2} \leq S_{1}+3$ while inserting $A_{1}$ after $A_{2}$ violates constraint $S_{2} \geq S_{1}+1$. This yields a solution to $\mathrm{P}_{1}$ displayed on Figure 3 having a makespan equal to 16 .

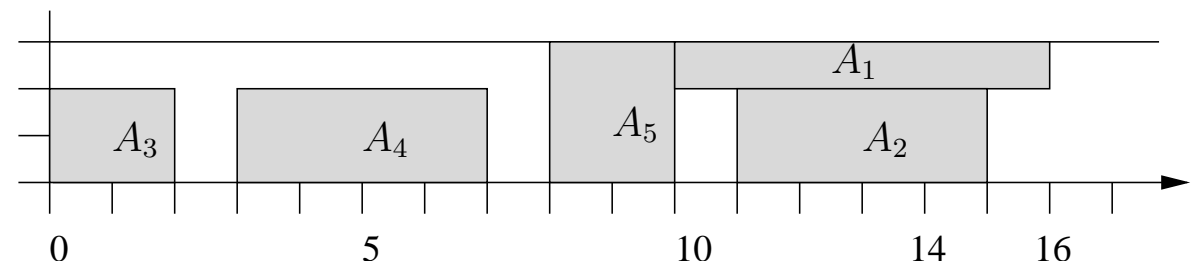

Fig. 3 Gantt chart of the solution to $\mathrm{P}_{1}$

\section{Feasible insertions}

Since flow $f$, feasible for problem $\left(P_{-x}\right)$, is an input data of insertion problem $\left(P_{x}\right)$, $\Delta_{i, j}(f), \mathcal{G}(f), F(f)$ and $L_{i j}(f)$ are simply denoted by $\Delta_{i, j}, \mathcal{G}, F$ and $L_{i j}$, respectively.

An insertion is defined as an ordered pair of disjoint activity sets $(\alpha, \beta)$ such that $(\alpha, \beta) \in V \backslash\left\{A_{x}, A_{n+1}\right\} \times V \backslash\left\{A_{0}, A_{x}\right\}$ and $\alpha \cap \beta=\emptyset$.

An insertion induces a set of arcs

$$
F(\alpha, \beta)=\left\{\left(A_{a}, A_{x}\right)\right\}_{A_{a} \in \alpha} \cup\left\{\left(A_{x}, A_{b}\right)\right\}_{A_{b} \in \beta} .
$$

We consider graph

$$
\mathcal{G}(\alpha, \beta)=G(V, E \cup F \cup F(\alpha, \beta), L(\alpha, \beta))
$$

where $L(\alpha, \beta)$ denotes the arc valuation induced by $(\alpha, \beta)$ and is defined as follows:

$$
L_{i j}(\alpha, \beta)= \begin{cases}p_{i} & \text { if } A_{i} \in \alpha, A_{j}=A_{x},\left(A_{i}, A_{x}\right) \notin E \\ \max \left(p_{i}, l_{i x}\right) & \text { if } A_{i} \in \alpha, A_{j}=A_{x},\left(A_{i}, A_{x}\right) \in E \\ p_{x} & \text { if } A_{i}=A_{x}, A_{j} \in \beta,\left(A_{x}, A_{j}\right) \notin E \\ \max \left(p_{x}, l_{x j}\right) & \text { if } A_{i}=A_{x}, A_{j} \in \beta,\left(A_{x}, A_{j}\right) \in E \\ L_{i j} & \text { if } A_{i} \in V \backslash \alpha, A_{j} \in V \backslash \beta\end{cases}
$$

Let $\Delta_{i, j}(\alpha, \beta)$ denotes the length of the longest path from $A_{i}$ to $A_{j}$ in $\mathcal{G}(\alpha, \beta)$. Let $Q_{k}(\alpha, \beta)$ denote the amount of resource $k$ units available for insertion in $(\alpha, \beta)$. Vector 
$Q(\alpha, \beta)$ is called the insertion capacity. We have

$$
Q_{k}(\alpha, \beta)=\sum_{A_{i} \in \alpha, A_{j} \in \beta} f_{i, j, k}
$$

We claim that any insertion $(\alpha, \beta)$ verifying

$$
Q_{k}(\alpha, \beta) \geq b_{x, k}, \forall R_{k} \in \mathcal{R}
$$

and such that there is no positive length cycle in $\mathcal{G}(\alpha, \beta)$ is feasible in the sense it yields at least a feasible solution $f^{\prime}$ to $\left(P_{x}\right)$.

In fact, a so-defined feasible insertion may yield several distinct feasible flows and Algorithm 1 (GENERATEFLOWFromInSERTION) computes one of them. Feasible flow $f^{\prime}$ is obtained by rerouting the flow sent from $\alpha$ to $\beta$. The algorithm runs in $O\left(n^{2} m\right)$. $Q_{k}$ denote at each inner step the total amount of flow already rerouted to $A_{x}$. Flow $f^{\prime}$ is feasible for $\left(P_{x}\right)$ if $(\alpha, \beta)$ verifies $(10)$ and if $\mathcal{G}\left(f^{\prime}\right)$ does not contain any positive length cycle. For the latter condition, we have $F\left(f^{\prime}\right) \subseteq F(\alpha, \beta) \cup F$. hence if $\mathcal{G}(\alpha, \beta)$ does not have any positive length cycle, $\mathcal{G}\left(f^{\prime}\right)$ does not have any positive length cycle neither and $\Delta_{0, n+1}\left(f^{\prime}\right) \leq \Delta_{0, n+1}(\alpha, \beta)$. Given a feasible insertion $(\alpha, \beta)$, only a subset of $\alpha$ may actually send flow to $A_{x}$ after insertion. Hence $\alpha$ is called the set of possible resource successors. Symmetrically, only a subset of $\beta$ may receive flow from $A_{x}$ and we call $\beta$ the set of possible resource successors. Note that differents orders for the enumeration of activities in $\alpha$ and $\beta$ (steps 3 and 3) may issue different flows.

Furthermore, given a flow $f^{\prime}$, solution to $P_{x}$ rerouted from $f$ through resource amounts $q_{i, j, k}$, an insertion $(\alpha, \beta)$ can be defined by setting $\alpha=\left\{A_{i} \in V \mid \exists A_{j} \in\right.$ $\left.V, \exists R_{k} \in \mathcal{R}, q_{i, j, k}>0\right\}$ and $\beta=\left\{A_{i} \in V \mid \exists A_{j} \in V, \exists R_{k} \in \mathcal{R}, q_{j, i, k}>0\right\} .(\alpha, \beta)$ is such that $\mathcal{G}(\alpha, \beta)=\mathcal{G}\left(f^{\prime}\right)$ and $\Delta_{0, n+1}\left(f^{\prime}\right) \leq \Delta_{0, n+1}(\alpha, \beta)$.

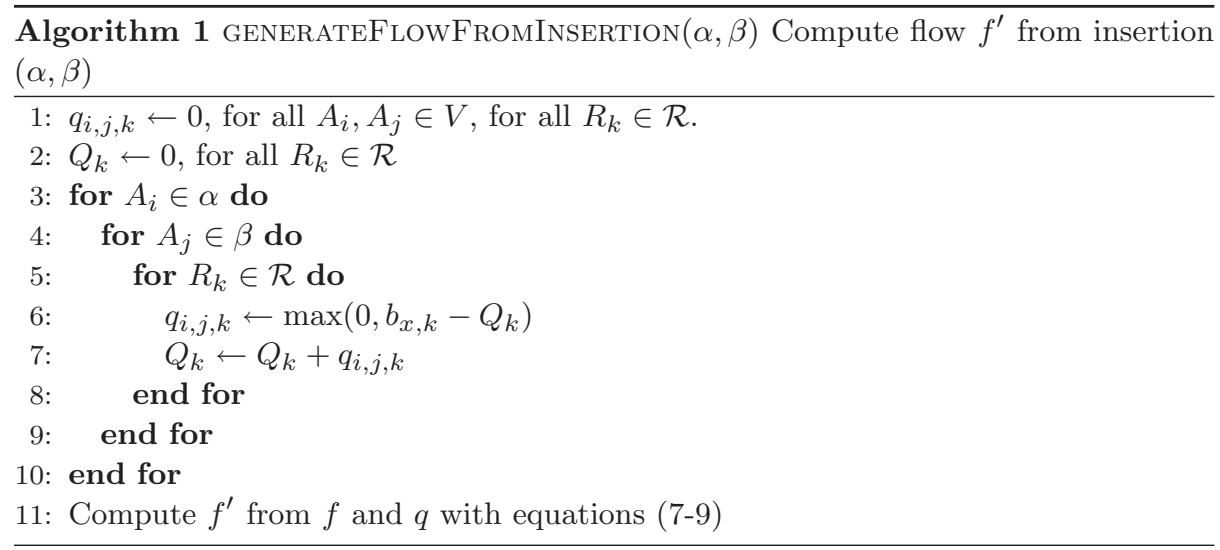

From this reduction, we can restrict the search space to pairs of activity sets $(\alpha, \beta)$ without considering explicitly amounts of rerouted flow $q_{i, j, k}$. It follows a simplified definition of the RCAIP.

Definition 4 (RCAIP) Given a solution flow $f$ to problem $\left(P_{-x}\right)$, the resourceconstrained activity insertion problem $\left(P_{x}\right)$ amounts to find an insertion (ordered pair of activity sets) $(\alpha, \beta)$ verifying capacity condition $(10)$, such that $\mathcal{G}(\alpha, \beta)$ does not include a positive length cycle and such that $\Delta_{0, n+1}(\alpha, \beta)$ is minimized. 


\section{Insertion feasibility conditions}

In this section, feasibility conditions of a given insertion $(\alpha, \beta)$ are established. The decision variant of $\left(P_{x}\right)$ is considered. The problem is to decide whether a feasible insertion with a makespan not greater than $v$ exists. The problem amounts to deciding whether there is a feasible solution to $\left(P_{x}\right)$ with $l_{(n+1) 0}=-v$. If $v<\Delta_{0, n+1}$, there is a positive length cycle in $\mathcal{G}$ and, consequently, the answer is no. In the remaining of this section, we assume $v \geq \Delta_{0, n+1}$.

Since only arcs from $F(\alpha, \beta)$ are added by the insertion of $A_{x}$, there are only three disjoint sets of elementary cycles absent from $\mathcal{G}$ that can be generated in $\mathcal{G}(\alpha, \beta)$.

- An elementary cycle belonging to set $\mathcal{C}_{1}(\alpha, \beta)$ involves an $\operatorname{arc}\left(A_{i}, A_{x}\right)$ with $A_{i} \in \alpha$ and $L_{i x}(\alpha, \beta)=p_{i}$ and no arc $\left(A_{x}, A_{j}\right)$ with $A_{j} \in \beta$. Note that in $\mathcal{G}(\alpha, \beta)$, a longest path from $A_{x}$ to $A_{i}$ which is not traversing any node of $\beta$ is also a longest path from $A_{x}$ to $A_{i}$ in $\mathcal{G}$ of length $\Delta_{x, i}$. Therefore, if $\mathcal{L}_{1}(\alpha, \beta)$ denotes the length of the longest cycles in $\mathcal{C}_{1}(v, \alpha, \beta)$, it follows that

$$
\mathcal{L}_{1}(\alpha, \beta)=\max _{A_{i} \in \alpha}\left(p_{i}+\Delta_{x, i}\right)
$$

- An elementary cycle belonging to set $\mathcal{C}_{2}(\alpha, \beta)$ includes an $\operatorname{arc}\left(A_{x}, A_{j}\right)$ with $A_{j} \in \beta$ and $L_{x j}(\alpha, \beta)=p_{x}$ and no arc $\left(A_{i}, A_{x}\right)$ with $A_{i} \in \alpha$. Using the same arguments as in the previous case, the length of the longest path from $A_{j}$ to $A_{x}$ in $\mathcal{G}(\alpha, \beta)$ is equal to $\Delta_{j, x}$. Consequently, the length of the longest cycle in $\mathcal{C}_{2}(\alpha, \beta)$ is

$$
\mathcal{L}_{2}(\alpha, \beta)=p_{x}+\max _{A_{j} \in \beta} \Delta_{j, x}
$$

- Last, an elementary cycle belonging to $\operatorname{set} \mathcal{C}_{3}(\alpha, \beta)$ involves two $\operatorname{arcs}\left(A_{i}, A_{x}\right),\left(A_{x}, A_{j}\right)$ with $L_{i x}(\alpha, \beta)=p_{i}$ and $L_{x j}(\alpha, \beta)=p_{x}$. Again, the longest path in $\mathcal{G}(\alpha, \beta)$ from $j$ to $i$ passes only through arcs existing in $\mathcal{G}$ and therefore is of length $\Delta_{j, i}$. Hence, the length of the longest cycle in $\mathcal{C}_{3}(\alpha, \beta)$ is

$$
\mathcal{L}_{3}(\alpha, \beta)=p_{x}+\max _{\left(A_{i}, A_{j}\right) \in \alpha \times \beta}\left(p_{i}+\Delta_{j, i}\right) .
$$

In what follows, $\mathcal{C}_{q}(\alpha, \beta)$ and $\mathcal{L}_{q}(\alpha, \beta)(q=1,2,3)$ will be simply denoted $\mathcal{C}_{q}$ and $\mathcal{L}_{q}$ when there is no ambiguity. Figure 4 illustrates the three considered cycle types.

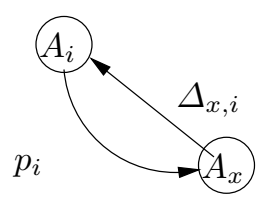

$\mathcal{C}_{1}$ cycle

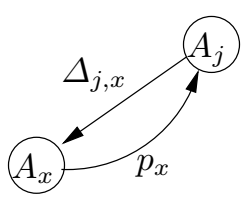

$\mathcal{C}_{2}$ cycle

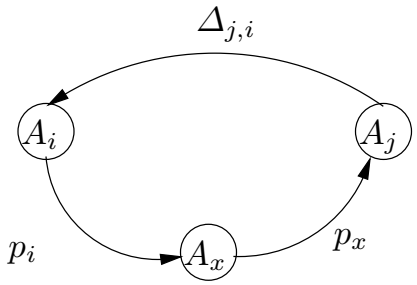

$\mathcal{C}_{3}$ cycle

Fig. 4 The three cycle types generated by the insertion

The feasibility condition for an insertion $(\alpha, \beta)$ becomes obvious from the characterization of the three possible cycle sets. 
Proposition 1 An insertion $(\alpha, \beta)$ is feasible for $\left(P_{x}\right)$ if and only if it verifies (10) and

$$
\max _{q=1,2,3} \mathcal{L}_{q}(\alpha, \beta) \leq 0
$$

\section{Computational complexity}

There have been previously established complexity results related to insertion. The activity insertion problem has already been addressed for the standard RCPSP in [1, $2]$. The problem is polynomial and an $\mathrm{O}\left(n^{2} m\right)$ optimal insertion procedure has been proposed by the authors. In [9], the authors study the job insertion problem in the standard job-shop scheduling problem and show the problem is NP-hard. In [8] and [10], a general insertion problem in disjunctive scheduling is defined. In disjunctive scheduling, resource constraints are defined through a set of disjunctive arcs $e=\{i, j\}$ such that, in any feasible solution called a selection, each disjunctive arc must be orientated yielding $\operatorname{arc}(i, j)$ or arc $(j, i)$. An insertion problem corresponds to a partial orientation (selection) of the disjunctive arcs and an insertion corresponds to a complete orientation (selection). Among other results, the authors show that when the insertion disjunctive graph has specific property, the insertion problem becomes solvable in polynomial time, in particular because feasible insertions correspond to independent sets in a bipartite graph. A disjunctive arc defines a set of activities that cannot be scheduled in parallel, i.e. a forbidden set of cardinality 2. The fundamental difference with the work of [9], [8] and [10] is that we cannot use the disjunctive graph formulation to represent our problem since in the RCPSP, the resource constraints define forbidden sets of cardinality that can be much larger than 2 . Hence, while insertion of single activities are generally easy in disjunctive graph models the following results show that this is not the case for our problem. Indeed, we establish the link between feasible insertions and independent sets in general graphs.

In what follows, we prove that the RCAIP is NP-hard. Hereafter, we define the decision variant of the RCAIP.

Definition 5 (RCAIP - decision variant) Given a solution flow $f$ to problem $\left(P_{-x}\right)$ and an integer $v$, does there exist an insertion $(\alpha, \beta)$ verifying conditions $(10-$ 11) and such that $\Delta_{0, n+1}(\alpha, \beta) \leq v ?$

We show that the decision problem is NP-complete by reduction from the independent set problem, defined below.

Definition 6 (INDEPENDENT SET - decision variant) Given a graph $G(\mathcal{V}, \mathcal{E})$, where $\mathcal{V}$ is a set of vertices and $\mathcal{E}$ is a set of edges, and an integer $k$, does there exist a subset of vertices $\mathcal{W} \subseteq \mathcal{V}$ of cardinality $k$ such that $\forall x, y \in \mathcal{W},(x, y) \notin \mathcal{E}$ (i.e. the vertices of $\mathcal{W}$ are pairwise independent) ?

Such a problem is known to be NP-complete [7]. Let $p$ denotes the number of vertices: $p=|\mathcal{V}|$.

Theorem 1 The decision variant of the RCAIP is NP-complete.

Proof The problem is in NP since (i) for any tentative solution $(\alpha, \beta)$ checking whether constraints (10) are satisfied can be made in $O\left(n^{2} m\right)$ time since $|\alpha| \leq n$ and $|\beta| \leq n$ 
and (ii) checking whether there is no positive length cycles in $\mathcal{C}_{1}, \mathcal{C}_{2}$ and $\mathcal{C}_{3}$ take $O(n)$, $O(n)$ and $O\left(n^{2}\right)$ time, respectively. Initial Matrix $\left(\Delta_{i, j}\right)$ can be computed in $O\left(n^{3}\right)$ by the Floyd-Warshall algorithm.

In what follows, we show how to associate to any independent set problem an instance of the RCAIP with $n=2 p+1$ non-dummy activities and a single resource of availability $B_{1}=p$ (the resource index will be omitted in the sequel).

The non-dummy activities are partitioned into three subsets $\Theta=\left\{A_{1}, \ldots, A_{p}\right\}$, $\Omega=\left\{A_{p+1}, \ldots, A_{2 p}\right\}$ and $\left\{A_{n}\right\}$ where $A_{n}=A_{2 p+1}$ is the activity to insert. All activities but $A_{n}$ are such that $b_{i}=p_{i}=1, \forall A_{i} \in \Theta \cup \Omega$. For $A_{n}$, we set $p_{n}=3$ and $b_{n}=k$. The resource flow $f$ is such that

$$
\begin{aligned}
f_{0, i}=1 & \forall A_{i} \in \Theta \\
f_{i, p+i}=1 & \forall A_{i} \in \Theta \\
f_{j, n+1}=1 & \forall A_{j} \in \Omega .
\end{aligned}
$$

All other resource flows are zero. The precedence constraints $E$ are such that there is an $\operatorname{arc}\left(A_{0}, A_{i}\right)$ with $l_{0 i}=0$, for each $A_{i} \in \Theta$. There is an arc $\left(A_{j}, A_{n+1}\right)$ with $l_{j(n+1)}=1$ for each $j \in \Omega$. There is an arc $\left(A_{i}, A_{p+i}\right)$ with $l_{i(p+i)}=2$ for each $A_{i} \in \Theta$. There is an arc $\left(A_{n+1}, A_{0}\right)$ such that $l_{(n+1) 0}=-5$ (i.e. the makespan has to be not greater than 5$)$. For the inserted task $A_{n}$, there is an arc $\left(A_{0}, A_{n}\right)$ and an arc $\left(A_{n}, A_{n+1}\right)$ with $l_{0 n}=0$ and $l_{n(n+1)}=3$, respectively.

The set of edges $\mathcal{E}$ of the graph of the independent set problem is arbitrarily orientated. Let $G(\mathcal{V}, \mathcal{U})$ denotes the graph so obtained. Now we create in $E$ an $\operatorname{arc}\left(A_{p+j}, A_{i}\right)$ with $l_{(p+j) i}=-3$ for each $(i, j) \in \mathcal{U}$ to represent the edges in $\mathcal{E}$. Note that the earliest schedule resulting from $f$ is optimal for $\left(P_{-n}\right)$ since $\Delta_{0, n+1}=\delta_{0, n+1}=3$.

Figure 5 shows how the insertion problem instance is built from an example graph of six nodes. Below each arc the time lag is indicated and the transferred resource flow is displayed between braces.

It can be easily checked that the minimal distance matrix $\Delta$ for the so-defined insertion problem instance (with $v=5$ ) is such that for each pair $A_{i}, A_{j}$ with $i \in \Theta$ and $j \in \Omega$, we have $\Delta_{j, i}=-3$ if $(j-p, i) \in \mathcal{U}$ and $\Delta_{j, i}=-4$ otherwise.

Below we show that there exists an insertion for $A_{n}$ in $\mathcal{G}$ if and only if there exists an independent set of cardinality $k$ in $G(\mathcal{V}, \mathcal{E})$. First, one can observe that if $W$ is an independent set of cardinality $k$, then there is a feasible insertion $(\alpha, \beta)$. Indeed, if $\alpha=\left\{A_{i} \mid i \in W\right\}$ and $\beta=\left\{A_{p+i} \mid i \in W\right\}$ then, because the flow from $A_{i} \in \Theta$ to $A_{i+p}$ is equal to 1 , we have $\sum_{A_{i} \in \alpha, A_{j} \in \beta} f_{i, j}=k$. Consequently $(\alpha, \beta)$ verifies capacity condition (10). Moreover, there is no maximum time lags involving the inserted activity $A_{n}$ and, consequently, no possible positive length cycles in $\mathcal{C}_{1}$ nor in $\mathcal{C}_{2}$. For any pair of nodes $x, y \in W$ we have no edge $(x, y)$ in $\mathcal{E}$, therefore we have no arc $(x+p, y)$ in $\mathcal{U}$. As a result, $\forall A_{j} \in \beta, \forall A_{i} \in \alpha$, we have $\Delta_{j, i}=-4$. The longest cycle in $\mathcal{C}_{3}$ has a length equal to $\mathcal{L}_{3}(\alpha, \beta)=\max _{\left(A_{i}, A_{j}\right) \in \alpha \times \beta}\left(p_{i}+p_{x}+\Delta_{j, i}\right)$. Since $p_{i}=1$ and $p_{x}=3$, we have $\mathcal{L}_{3}(\alpha, \beta)=0$. It follows that the insertion problem is feasible.

Now let us show that if the insertion is feasible, there is an independent set of cardinality $k$. Let $(\alpha, \beta)$ denotes the feasible insertion. Any insertion such that $\alpha=$ $\left\{A_{0}\right\}$ and $\beta \subseteq \Theta \neq \emptyset$ is not feasible since it will increase the project duration to 6 , whereas it is bounded by 5 . The same remark holds for the insertion such that $\alpha \subseteq \Omega$ and $\beta=\left\{A_{n+1}\right\}$. Therefore, $A_{n}$ can only be located between some activities of $\Theta$ and some activities of $\Omega$ (i.e. $\alpha \subseteq \Theta$ and $\beta \subseteq \Omega$ ). For a feasible $(\alpha, \beta)$ insertion, 

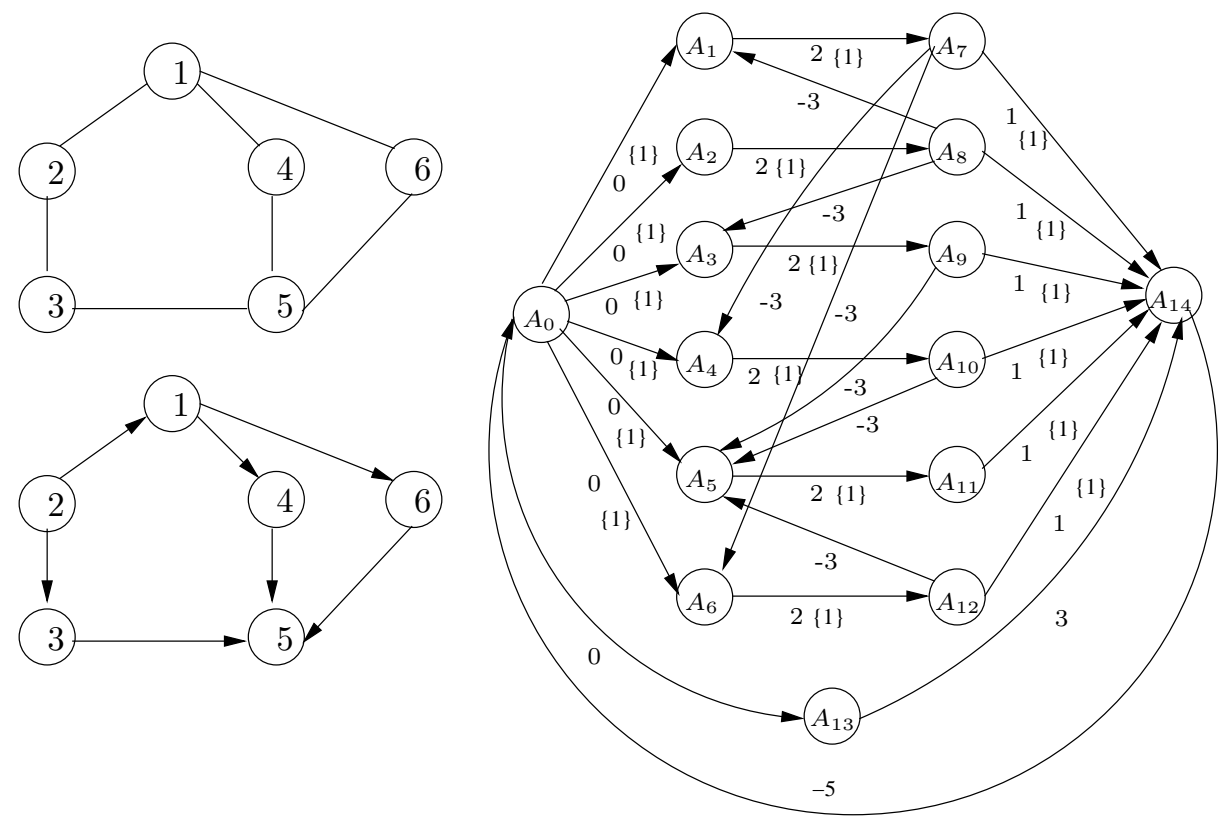

Fig. 5 Reduction from independent set

since no positive length cycles can generated, we have $\Delta_{j, i}=-4 \forall A_{i} \in \alpha, \forall A_{j} \in \beta$. Subsequently there is no $\operatorname{arc}(j-p, i)$ in $\mathcal{U}$ and no edge $(j-p, i)$ in $E, \forall A_{i} \in \alpha$, $\forall A_{j} \in \beta$. Furthermore, since the insertion is feasible, we have $|\alpha|=k$ therefore $\alpha$ is an independent set of cardinality $k$. Figure 6 illustrates the correspondence between an independent set (bold nodes) and an insertion.
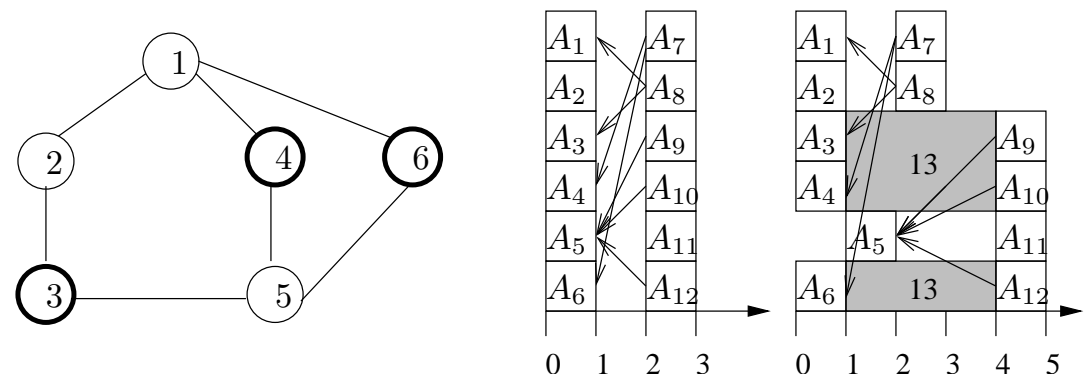

Fig. 6 A feasible independent set and its corresponding feasible insertion

While for the standard RCPSP, Artigues et al. proposed a polynomial algorithm to solve the insertion problem $[1,2]$, the introduction of minimum and maximum time lags clearly makes the problem intractable despite of its apparent simplicity. This illustrates the deep modifications of the problem structure brought by the maximum time lags. To confirm this, we exhibit in the following section a polynomial algorithm, able to solve the insertion problem when only minimum time lags are considered. 


\section{RCAIP with minimum time lags only}

We suppose in this section that all time lags are non-negative and that $p_{i} \geq 1, \forall A_{i} \in \mathcal{A}$. We still assume that $f$ represents a feasible solution to $\left(\mathrm{P}_{-x}\right)$ of makespan $\Delta_{0, n+1}$ corresponding to the length of the longest path between 0 and $n+1$ in $\mathcal{G}$.

We already know that the insertion problem is polynomial for the standard RCPSP as stated in [2]. The standard RCPSP corresponds to the case where all minimum time lags are such that $l_{i j}=p_{i}$. it follows that in the case where all minimum time lags are such that $l_{i j} \geq p_{i}$, we can trivially show that the problem is polynomially solvable by transforming the RCPSP instance with minimum time lags into a standard RCPSP instance as follows. For each $\left(A_{i}, A_{j}\right) \in E$, create an activity $A_{i j}$ of duration $l_{i j}-p_{i}$ and zero resource demand and augment $E$ with the precedence constraints $\left(A_{i}, A_{i j}\right)$ and $\left(A_{i j}, A_{j}\right)$. Then, set all arc values to the duration of the origin activity. Since the initial flow is trivially valid for the so-generated instance, the polynomial insertion algorithm for the standard RCPSP can be applied. Thus, the problem remains open only in the case there is at least one minimum time lag such that $0 \leq l_{i j} \leq p_{i}$. We show that in this case the insertion problem $\left(P_{x}\right)$ remains polynomially solvable. We now consider the optimization variant, aiming at finding a minimal makespan feasible insertion (so we have $l_{(n+1) 0}=-\infty$ ).

\subsection{Makespan after insertion}

Given, a feasible insertion $(\alpha, \beta)$, the makespan $\Delta_{0, n+1}(\alpha, \beta)$ after insertion in $(\alpha, \beta)$ is either equal to the makespan before insertion $\Delta_{0, n+1}$ or to the length of the longest path from $A_{0}$ to $A_{n+1}$ in $\mathcal{G}(\alpha, \beta)$ traversing node $A_{x}$. This corresponds to three possible sets of $(0, n+1)$ paths.

- The set of paths traversing an $\operatorname{arc}\left(A_{i}, A_{x}\right)$ with $\left(A_{i} \in \alpha\right)$ and no $\operatorname{arc}\left(A_{x}, A_{j}\right)$ with $\left(A_{j} \in \beta\right)$. The longest path of this set has a length equal to

$$
\mathcal{M}_{1}(\alpha, \beta)=\max _{A_{i} \in \alpha}\left(\Delta_{0, i}+p_{i}\right)+\Delta_{x, n+1}
$$

- The set of paths traversing an $\operatorname{arc}\left(A_{x}, A_{j}\right)$ with $\left(A_{j} \in \beta\right)$ and no $\operatorname{arc}\left(A_{i}, A_{x}\right)$ with $\left(A_{i} \in \alpha\right)$. The longest path of this set has a length equal to

$$
\mathcal{M}_{2}(\alpha, \beta)=\Delta_{0, x}+p_{x}+\max _{A_{j} \in \beta}\left(\Delta_{j, n+1}\right)
$$

- The set of paths traversing an $\operatorname{arc}\left(A_{i}, A_{x}\right)$ with $\left(A_{i} \in \alpha\right)$ and an $\operatorname{arc}\left(A_{x}, A_{j}\right)$ with $\left(A_{j} \in \beta\right)$. The longest path of this set has a length equal to

$$
\mathcal{M}_{1}(\alpha, \beta)=\max _{A_{i} \in \alpha}\left(\Delta_{0, i}+p_{i}\right)+p_{x}+\max _{A_{j} \in \beta}\left(\Delta_{j, n+1}\right)
$$

Figure 7 illustrates the 3 types of $(0, n+1)$ paths.

It follows that the makespan of a feasible insertion $(\alpha, \beta)$ is equal to

$$
\Delta_{0, n+1}(\alpha, \beta)=\max \left(\Delta_{0, n+1}, \mathcal{M}_{1}(\alpha, \beta), \mathcal{M}_{2}(\alpha, \beta), \mathcal{M}_{3}(\alpha, \beta)\right)
$$




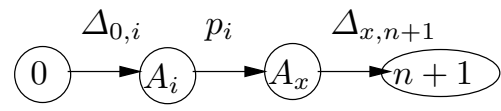

type 1 path

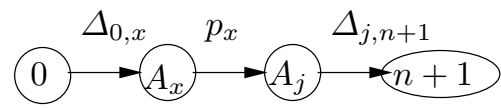

type 2 path

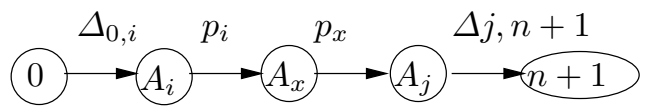

type 3 path

Fig. 7 The 3 types of $(0, n+1)$ paths generated by the insertion

6.2 Feasibility conditions and feasible insertion

Below, we a necessary and sufficient condition for the existence of a feasible insertion is derived. In the case the condition is verified, a feasible insertion is provided.

Let $\gamma$ denote the set of non-dummy activities linked with $A_{x}$ by a synchronization constraint, i.e.:

$$
\gamma=\left\{A_{i} \in \mathcal{A} \mid \Delta_{i, x}=0 \text { and } \Delta_{x, i}=0\right\}
$$

Note that $A_{n+1} \notin \gamma$ since $A_{n+1}$ cannot be synchronized with $A_{x}$. Indeed, $l_{x, n+1}=$ $p_{x} \geq 1$. By convention, even if have $l_{0, x}=l_{x, 0}=0$, we assume that dummy activity $A_{0}$ does not belong to $\gamma$. Consequently, for any activity $A_{i} \in \gamma$ we cannot include $A_{i}$ in the set of possible predecessors $\alpha$ (otherwise a cycle of length $p_{a}$ would be issued) nor in the set of possible successors $\beta$ (otherwise a cycle of length $p_{x}$ would be issued). Furthermore, in any feasible schedule respecting the precedence constraints, the synchronized activities must start exactly at the same time. Hence, there exists a solution to the RCAIP only if the following inequalities hold.

$$
\sum_{A_{i} \in \gamma} b_{i, k}+b_{x, k} \leq B_{k} \quad \forall R_{k} \in \mathcal{R}
$$

In fact, (13) is a necessary and sufficient feasibility condition for the RCAIP. Indeed, insertion $\left(\alpha_{0}, \beta_{0}\right)$ defined hereafter is feasible if and only if (13) hold, which will be shown through Lemma 1.

Let $\alpha_{0}$ denote the set of activities $A_{i}$ which are not synchronized with $A_{x}$ and such that there is a non negative length path from $A_{i}$ to $A_{x}$.

$$
\alpha_{0}=\left\{A_{i} \in V \backslash \gamma \mid \Delta_{i, x} \geq 0\right\}
$$

Let $\beta_{0}$ denote the complement set of $\alpha_{0}$ in $V$ minus the activities synchronized with $A_{x}$.

$$
\beta_{0}=V \backslash\left(\gamma \cup \alpha_{0}\right)
$$

Lemma 1 The RCAIP is feasible and $\left(\alpha_{0}, \beta_{0}\right)$ is a feasible insertion if and only if $\gamma$ satisfies constraints (13). 
Proof First we show that $\left(\alpha_{0}, \beta_{0}\right)$ verifies capacity condition (10) if and only if $\gamma$ satisfies constraints (13). Consider insertion $\left(\alpha^{0}, \beta^{0}\right)=\left(\left\{A_{0}\right\}, V \backslash\left\{A_{0}\right\}\right)$. Due to flow conservation of $A_{0}$ we have $Q_{k}\left(\alpha^{0}, \beta^{0}\right)=B_{k}$. Consider the activities of $V \backslash\left\{A_{0}\right\}$ such that $\Delta_{i, x} \geq 0$. Assume these activities $\left\{A_{i_{1}}, \ldots, A_{i_{w}}\right\}$ are sorted in decreasing order of $\Delta_{i_{v}, x}, v=1, \ldots, w$. Consider now insertions $\left(\alpha^{v}, \beta^{v}\right), v=1, \ldots, w$, such that $\left(\alpha^{v}, \beta^{v}\right)=\left(\alpha^{v-1} \cup\left\{A_{i_{t}}\right\}, \beta^{v-1} \backslash\left\{A_{i_{v}}\right\}\right)$. In other words, $\left(\alpha^{v}, \beta^{v}\right)$ is obtained by moving $A_{i_{v}}$ from $\beta^{v-1}$ to $\alpha^{v-1}$. Since $\Delta_{i_{v}, x} \leq \Delta_{i_{v-1}, x}$, all the flow received by $A_{i_{v}}$ is received only from $\left\{A_{0}, \ldots, A_{i_{v-1}}\right\}=\alpha^{v-1}$ and all the flow sent from $A_{i_{v}}$ is sent to $V \backslash\left\{A_{0}, \ldots, A_{i_{v-1}}\right\}=\beta^{v-1}$. Consequently $Q_{k}\left(\alpha^{v}, \beta^{v}\right)=Q_{k}\left(\alpha^{v-1}, \beta^{v-1}\right)=B_{k}$, for all $R_{k} \in \mathcal{R}$ and for all $v=1, \ldots, w$.

Observe now that $\left(\alpha_{0}, \beta_{0}\right)$ is obtained from $\left(\alpha^{w}, \beta^{w}\right)$ by removing the activities synchronized with $A_{x}$ (set $\left.\gamma\right)$ from the set of possible predecessors $\alpha^{w}$. Since $\Delta_{i, x}=0$ for any activity $A_{i} \in \gamma$ we have $\Delta_{i, x} \leq \Delta_{i_{v}, x}$ for all $v=1, \ldots, w$ and so any activity belonging to $\gamma$ can only send flow to activities of $V \backslash\left\{A_{0}, \ldots, A_{i_{w}}\right\}=\beta^{w}$. Hence, we have

$$
Q_{k}\left(\alpha_{0}, \beta_{0}\right)=B_{k}-\sum_{i \in \gamma} b_{i, k}, \forall R_{k} \in \mathcal{R}
$$

Consequently if $\gamma$ verifies (13), capacity condition (10) is verified by $\left(\alpha_{0}, \beta_{0}\right)$.

Second, we show the length of cycles $\mathcal{C}_{q}\left(\alpha_{0}, \beta_{0}\right), q=1,2,3$ are non positive. From the definition of $\alpha_{0}$, there is no path in $\mathcal{G}$ from $x$ to any activity $A_{i} \in \alpha_{0}$. It yields $\Delta_{x, i}=-\infty$ and $\mathcal{L}_{1}\left(\alpha_{0}, \beta_{0}\right)=-\infty$. From the definition of $\beta_{0}$, there is no path in $\mathcal{G}$ from any activity $A_{j} \in \beta_{0}$ and $x$. Therefore, $\Delta_{j, x}=-\infty$ and $\mathcal{L}_{2}\left(\alpha_{0}, \beta_{0}\right)=-\infty$. There is also no path in $\mathcal{G}$ from any activity $A_{j} \in \beta_{0}$ and any activity $A_{i} \in \alpha_{0}$, since otherwise $\Delta_{j, x} \geq 0$. Consequently, $\Delta_{j, i}=-\infty$ and $\mathcal{L}_{3}\left(\alpha_{0}, \beta_{0}\right)=-\infty$.

Condition (13) for the existence of a feasible insertion can be checked in $O(\mathrm{~nm})$. Feasible insertion $\left(\alpha_{0}, \beta_{0}\right)$ can be obtained in $O(|E \cup F|)$ since checking if $\Delta_{i, x} \geq 0$ can be done by a simple depth-first search algorithm in $\mathcal{G}$.

\subsection{Dominant insertions}

Let $(\alpha, \beta)$ denotes a feasible insertion. Let $\mu(\alpha)$ denotes the subset of $\alpha$ such that

$$
\mu(\alpha)=\left\{A_{i} \in \alpha \mid \Delta_{0, i}+p_{i}=\max _{A_{a} \in \alpha}\left(\Delta_{0, a}+p_{a}\right)\right\}
$$

and $\nu(\beta)$ denotes the subset of $\beta$ such that

$$
\nu(\beta)=\left\{A_{i} \in \beta \mid \Delta_{i, n+1}=\max _{A_{b} \in \beta}\left(\Delta_{b, n+1}\right)\right\} .
$$

$\mu(\alpha)$ is the set of activities of $\alpha$ yielding the longest path of length $\mathcal{M}_{1}(\alpha, \beta) . \nu(\beta)$ is the set of activities of $\beta$ yielding the longest path of length $\mathcal{M}_{2}(\alpha, \beta) .\{\mu(\alpha), \nu(\beta)\}$ is the pair of activity subsets yielding the longest path of length $\mathcal{M}_{3}(\alpha, \beta)$. Thus, due to the expression of the makespan after insertion (12), to obtain an insertion $\left(\alpha^{\prime}, \beta^{\prime}\right)$ of makespan lower than $\Delta_{0, n+1}(\alpha, \beta)$, we must have $\mu(\alpha) \cap \alpha^{\prime}=\emptyset$ or $\nu(\beta) \cap \beta^{\prime}=\emptyset$. For any $A_{i} \in \mu(\alpha)$ and $A_{j} \in \nu(\beta)$, we conclude that $(\alpha, \beta)$ is dominant compared to any other insertion $\left(\alpha^{\prime}, \beta^{\prime}\right)$ verifying $A_{i}$ belongs to the set of possible predecessors $\alpha^{\prime}$ and $A_{j}$ belongs to the set of possible successors $\beta^{\prime}$. Lemma 2 below shows that from $\left(\alpha_{0}, \beta_{0}\right)$, we can derive an insertion with even stronger dominance properties. 
Given an activity set $e$, consider the intermediate insertion problem $P_{x}(e)$ aiming at finding the optimal insertion $(\alpha, \beta)$ such that $\beta \cap e \neq \emptyset$, i.e. such that at least one possible successor belongs to $e$. Suppose activities of $\alpha_{0}$ are sorted in increasing $\Delta_{0, i}+p_{i}$ such that $\alpha_{0}=\left\{A_{i_{1}}, \ldots, A_{i_{\left|\alpha_{0}\right|}}\right\}$. Let $\alpha_{0}^{*}$ denote the smallest subset $\left\{A_{i_{1}}, \ldots, A_{i_{\left|\alpha_{0}^{*}\right|}}\right\} \subseteq$ $\alpha_{0}$ such that $Q\left(\alpha_{0}^{*}, \beta_{0}\right) \geq b_{x k}$ for all $R_{k} \in \mathcal{R}$.

Lemma $2\left(\alpha_{0}^{*}, \beta_{0}\right)$ is the optimal solution of $P_{x}\left(\nu\left(\beta_{0}\right)\right)$.

Proof We already know that $\left(\alpha_{0}, \beta_{0}\right)$ dominates any insertion $(\alpha, \beta)$ such that $\mu\left(\alpha_{0}\right) \cap$ $\alpha \neq \emptyset$ and $\nu\left(\beta_{0}\right) \cap \beta \neq \emptyset$. By construction, $\left(\alpha_{0}^{*}, \beta_{0}\right)$ dominates any insertion $(\alpha, \beta)$ such that $\alpha \subseteq \alpha_{0}$ and $\nu\left(\beta_{0}\right) \cap \beta \neq \emptyset$. The case $\alpha \cap \beta_{0} \neq \emptyset$ remains to be checked. Consider an insertion $(\alpha, \beta)$ with $\nu\left(\beta_{0}\right) \cap \beta \neq \emptyset, \alpha \cap \beta_{0} \neq \emptyset$ and $\Delta_{0, n+1}(\alpha, \beta)<\Delta_{0, n+1}\left(\alpha_{0}^{*}, \beta_{0}\right)$. Let $e=\alpha \cap \beta_{0}$ denote the set of activities of $\alpha$ that belong also to $\beta_{0}$. Suppose in addition that $e=\left\{A_{i_{1}}, \ldots, A_{i_{|e|}}\right\}$ is sorted in increasing $\Delta_{0, i_{v}}+p_{i_{v}}$ and that $\Delta_{0, i_{|e|}}+p_{i_{|e|}}$ is minimal. Then, there is no flow sent from $A_{i_{|e|}}$ to $A_{i_{v}}, v<|e|$. There is no flow sent from $A_{i_{|e|}}$ to any activity of $\alpha \backslash e$ neither, otherwise we would have $\Delta_{i_{|e|}, x} \geq 0$ and thus $A_{i_{|e|}} \in \alpha_{0}$. Hence, all the flow sent by $A_{i_{|e|}}$ is received by $\beta_{0}$ and we have $Q_{k}\left(\left\{A_{i_{|e|}}\right\}, \beta_{0}\right)=b_{i_{|e|}, k}$, for all $R_{k} \in \mathcal{R}$. Let $\Gamma\left(A_{i_{|e|}}\right)$ denote the set of activities sending flow to $A_{i_{|e|}}$ (its resource predecessors). Since $A_{i_{|e|}} \in \alpha, \Gamma\left(A_{i_{|e|}}\right)$ can be included in $\alpha$ without generating any cycle. Also, $A_{i_{|e|}}$ can be moved from $\alpha$ to $\beta$. without generating any cycle since $A_{i_{|e|}} \in \beta_{0}$ and there is no path from $A_{i_{|e|}}$ to $i_{v}$, $v<|e|$. By performing both operations, the insertion capacity cannot decrease and the insertion is still feasible. Furthermore, all activities $A_{j}$ in $\Gamma\left(A_{i_{|e|}}\right)$ are such that $\Delta_{0, j}+p_{j}<\Delta_{0, i_{|e|}}+p_{|e|}$ since $p_{j} \geq 1$ for all $j$, which contradicts the minimality of $\Delta_{0, i_{|e|}}+p_{i_{|e|}}$

Lemma 2 shows that $\left(\alpha_{0}^{*}, \beta_{0}\right)$ dominates any other insertion $(\alpha, \beta)$ such that at least one activity in $\nu\left(\beta_{0}\right)$ belongs to the set of possible successors $\beta$. Thus, we may now consider only insertions such that $\nu\left(\beta_{0}\right) \cap \beta=\emptyset$. In set $\nu\left(\beta_{0}\right)$ some activities are possible resource predecessors while other activities are not. Let $\nu^{\prime}(\beta)$ denote the subset of $\nu(\beta)$ such that $\Delta_{x, i}=-\infty$, for all $A_{i} \in \nu^{\prime}(\beta)$. All activities in $\nu^{\prime}\left(\beta_{0}\right)$ are possible resource predecessors for $A_{x}$. We define a series of insertions $\left(\alpha_{t}, \beta_{t}\right)_{t \geq 0}$ starting from $\left(\alpha_{0}, \beta_{0}\right)$ and performing recurrent modifications as follows:

$$
\left(\alpha_{t+1}, \beta_{t+1}\right)=\left(\alpha_{t} \cup \nu^{\prime}\left(\beta_{t}\right), \beta_{t} \backslash \nu\left(\beta_{t}\right)\right)
$$

Lemma $3\left(\alpha_{t+1}, \beta_{t+1}\right)$ is feasible if and only if $\left(\alpha_{t}, \beta_{t}\right)$ is feasible and $Q_{k}\left(\alpha_{t+1}, \beta_{t+1}\right) \geq b_{x, k}$, for all $R_{k} \in \mathcal{R}$.

Proof Since $\left(\alpha_{t}, \beta_{t}\right)$ is feasible, we have to show that no positive length cycle is generated by the operation performed on $\left(\alpha_{t}, \beta_{t}\right)$ to compute $\left(\alpha_{t+1}, \beta_{t+1}\right)$. Obviously, removing $\nu\left(\beta_{t}\right)$ from $\beta_{t}$ cannot increase any cycle length. Since $\left(\alpha_{t}, \beta_{t}\right)$ is feasible, no cycle can be added in $\mathcal{C}_{2}\left(\alpha_{t+1}, \beta_{t+1}\right)$. Since $\Delta_{x, i}=-\infty$, for all $A_{i} \in \nu^{\prime}\left(\beta_{t+1}\right)$, no cycle is added in $\mathcal{C}_{1}\left(\alpha_{t+1}, \beta_{t+1}\right)$. Since $\Delta_{i, n+1}=\max _{A_{b} \in \beta_{t}}\left(\Delta_{b, n+1}\right)$, for all $A_{i} \in \nu^{\prime}\left(\beta_{q}\right)$, there is no path from $A_{j} \in \beta_{t} \backslash \nu\left(\beta_{t}\right)$ to $A_{i}$ and no cycle is added in $\mathcal{C}_{3}\left(\alpha_{t+1}, \beta_{t+1}\right)$. Hence $\left(\alpha_{t+1}, \beta_{t+1}\right)$ remains feasible if its resource capacity remains sufficient.

If $\alpha_{t}$ is feasible, suppose $\alpha_{t}=\left\{i_{1}, \ldots, i_{\left|\alpha_{t}\right|}\right\}$ where activities $i_{v}$ are sorted in increasing $\Delta_{0, i_{t}}+p_{i_{v}}$. Let $\alpha_{t}^{*}$ denote the smallest subset $\left\{i_{1}, \ldots, i_{\left|\alpha_{t}^{*}\right|}\right\}$ of $\alpha_{t}$ such that $Q\left(\alpha_{t}^{*}, \beta_{t}\right) \geq b_{x, k}$ for all $R_{k} \in \mathcal{R}$. 
Note that since set $\nu\left(\beta_{t}\right)$ contains at least one activity, there are at most $n$ terms in the series until $\beta_{t}$ becomes empty. Suppose insertions $\left(\alpha_{t}, \beta_{t}\right)_{0 \leq t<\rho}$ are feasible while $\left(\alpha_{\rho}, \beta_{\rho}\right)$ is infeasible.

Lemma 4 Each feasible insertion $\left(\alpha_{t}^{*}, \beta_{t}\right)$ is an optimal solution of $P_{x}\left(\nu\left(\beta_{t}\right)\right)$ and all solutions $(\alpha, \beta)$ such that $\beta \cap \beta_{\rho} \neq \emptyset$ are dominated by one solution $\left(\alpha_{t}^{*}, \beta_{t}\right), t<\rho$.

Proof If $\left(\alpha_{t}, \beta_{t}\right)$ is feasible, the same arguments as for lemma 2 can be used to show that $\left(\alpha_{t}^{*}, \beta_{t}\right)$ is the optimal solution of $P_{x}\left(\nu\left(\beta_{t}\right)\right)$. If $\left(\alpha_{\rho}, \beta_{\rho}\right)$ is not feasible, we have a resource $R_{k} \in \mathcal{R}$ such that $Q_{k}\left(\alpha_{\rho}, \beta_{\rho}\right)<b_{x, k}$ (from lemma 3 ). Hence the flow sent from $\alpha_{\rho}$ to $\beta_{\rho}$ is not sufficient to insert $A_{x}$. Consequently, some activities of $\alpha_{\rho}$ should appear as possible successors in $\beta$ in any feasible solution such $(\alpha, \beta)$ that $\beta \cap \beta_{\rho} \neq \emptyset$. Any possible successor in $\alpha_{\rho}$ belongs also to a set $\nu\left(\beta_{t}\right), t<\rho$ and so $(\alpha, \beta)$ is dominated by $\left(\alpha_{t}, \beta_{t}\right)$

Theorem 2 The series $\left(\alpha_{t}^{*}, \beta_{t}\right)_{0 \leq t<\rho}$ is dominant.

Proof The property follows from lemma 4. Any activity $A_{i}$ belongs to one of the following sets $\alpha_{0}, \gamma, \nu\left(\beta_{t}\right)_{0 \leq t<\rho}$ or $\beta_{\rho}$. If $A_{i}$ belongs to $\alpha_{0}$ or to $\gamma, A_{i}$ is not a possible successor of $A_{x}$. If $A_{i}$ belongs to $\nu\left(\beta_{t}\right)$ for some $t \in\{0, \ldots, \rho-1\}$, the optimal solution $(\alpha, \beta)$ with $A_{i} \in \beta$ is included in the series. If $A_{i}$ is in $\beta_{\rho}$ then any insertion such that $A_{i} \in \beta$ is dominated by one insertion of the series.

\subsection{Polynomial insertion algorithm}

We propose Algorithm 2 (OptInsERT) generating the series of dominant insertions in polynomial time. Set $\gamma$ of synchronized activities is computed at step 1 . The feasibility condition is checked at step 1 . It problem $\left(P_{x}\right)$ is feasible, the initial insertion $\left(\alpha_{0}, \beta_{0}\right)$ is computed at steps 3-4. Loop 6-17 generates insertion series $\left(\alpha_{t}, \beta_{t}\right)$ starting from $\left(\alpha_{0}, \beta_{0}\right)$. Loop 8-11 compute set $\alpha_{t}^{*}$. Steps 12-16 compute $\left(\alpha_{t+1}, \beta_{t+1}\right)$ from $\left(\alpha_{t}, \beta_{t}\right)$, $\nu$ and $\nu^{\prime}$. Finally, Step 18 retrieves the optimal insertion and Step 19 compute the optimal flow. The algorithm can be implemented easily in $O\left(n^{2} m\right)$ time.

\subsection{Illustrative example}

Finally, this section illustrates the behavior of the algorithm. Let us consider a RCPSP example with 10 activities and two resources such that $B_{1}=7$ and $B_{2}=4$. The processing times and the resource demands are indicated on Table 2. Minimum time lags are displayed on Figure 8. We assume that all activities, except Activity $A_{5}$ that has to be inserted, are already scheduled as shown on the Gantt diagram of Figure 10. The flow network $f$ corresponding to this partial schedule is displayed on Figure 9 and gives a makespan $C_{\max }=11$. The arc weight $L_{i j}$ and flow values $f_{i, j, k}$ (between parenthesis) are displayed near each arc. In the resource flow network, $A_{5}$ is isolated.

The values of $\Delta_{0, i}+p_{i}$ and $\Delta_{i, n+1}$, for all activities $A_{i}$, needed to check the feasibility or compute the makespan of any insertion can be computed using the BellmannFord's algorithm. They are indicated in Table 3.

Sets $\gamma, \alpha_{0}$ and $\beta_{0}$ are defined as follows. $\gamma=\{6\}$ since $A_{6}$ is the only activity which is synchronized with activity $5\left(S_{5}=S_{6}\right)$. Then we find $\alpha_{0}=\left\{A_{0}, A_{2}, A_{3}, A_{4}\right\}$ 


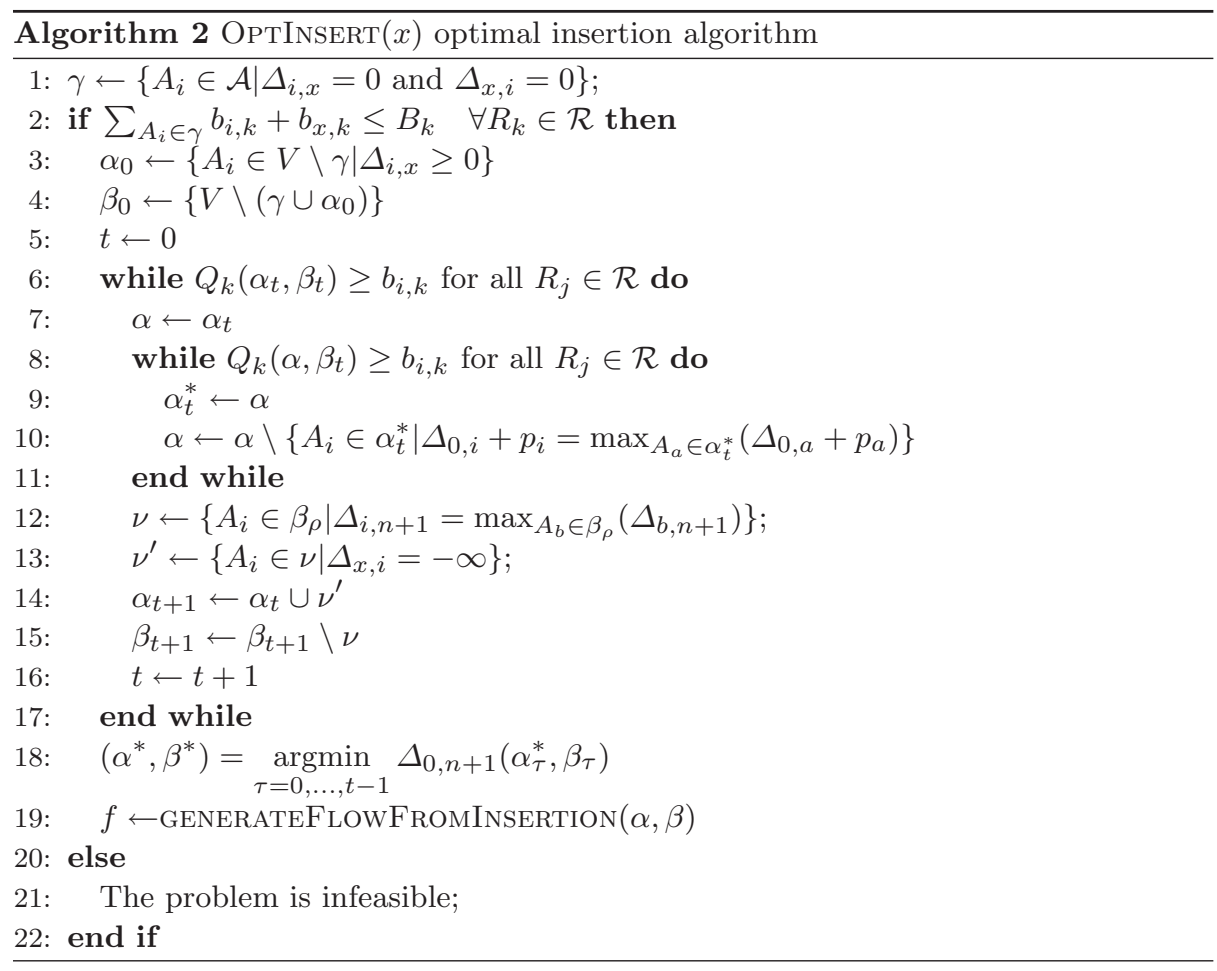

Table 2 Processing times and resource demands

\begin{tabular}{lcccccccccc}
\hline$i$ & 1 & 2 & 3 & 4 & 5 & 6 & 7 & 8 & 9 & 10 \\
$p_{i}$ & 6 & 1 & 1 & 2 & 5 & 3 & 5 & 3 & 2 & 4 \\
$\left(b_{i, 1}, b_{i, 2}\right)$ & $(2,1)$ & $(1,0)$ & $(3,1)$ & $(2,0)$ & $(1,2)$ & $(2,1)$ & $(2,0)$ & $(1,1)$ & $(1,2)$ & $(1,1)$ \\
\hline
\end{tabular}

Table 3 Values of $\Delta_{0, i}+p_{i}$ and $\Delta_{i, n+1}$

\begin{tabular}{lcccccccccccc}
\hline$i$ & 0 & 1 & 2 & 3 & 4 & 5 & 6 & 7 & 8 & 9 & 10 & 11 \\
$\Delta_{0, i}+p_{i}$ & 0 & 7 & 1 & 1 & 2 & 6 & 4 & 10 & 7 & 5 & 11 & 11 \\
$\Delta_{i, n+1}$ & 11 & 10 & 11 & 11 & 9 & 8 & 8 & 5 & 3 & 6 & 4 & 0 \\
\hline
\end{tabular}

and $\beta_{0}=\left\{A_{1}, A_{7}, A_{8}, A_{9}, A_{10}, A_{11}\right\}$ (see the cut denoted $t=0$ on Figure 9). This insertion $\left(\alpha_{0}, \beta_{0}\right)$ is resource-feasible since, $B_{1}-b_{6,1}=5 \geq b_{5,1}=1$ and $B_{2}-b_{6,2}=$ $3 \geq b_{5,2}=2$. We may check in Table 3 that $\mu\left(\alpha_{0}, \beta_{0}\right)=\left\{A_{4}\right\}$ and $\nu\left(\alpha_{0}, \beta_{0}\right)=\left\{A_{1}\right\}$ with $\Delta_{0,4}+p_{4}=2$ and $\Delta_{1,11}=10$. The insertion yields a makespan

$$
\Delta_{0, n+1}(\alpha, \beta)=\max \left(\Delta_{0, n+1}, \mathcal{M}_{1}\left(\alpha_{0}, \beta_{0}\right), \mathcal{M}_{2}\left(\alpha_{0}, \beta_{0}\right), \mathcal{M}_{3}\left(\alpha_{0}, \beta_{0}\right)\right)=17 .
$$

since $\Delta_{0, n+1}=11, \mathcal{M}_{1}\left(\alpha_{0}, \beta_{0}\right)=\Delta_{0,4}+p_{4}+\Delta_{5,11}=10, \mathcal{M}_{2}\left(\alpha_{0}, \beta_{0}\right)=\Delta_{0,5}+p_{5}+$ $\Delta_{1,11}=16$ and $\mathcal{M}_{3}\left(\alpha_{0}, \beta_{0}\right)=\Delta_{0,4}+p_{4}+p_{5}+\Delta_{1,11}=17 .\left(\alpha_{0}, \beta_{0}\right)$ is an optimal insertion among all insertion such that $A_{4}$ is a resource predecessor and $A_{1}$ is a resource successor.

We now give the dominant insertions computed by Algorithm 2. For $t=0$, dominant insertion $\left(\alpha_{0}^{*}, \beta_{0}\right)$ is computed by removing as many activities $A_{i}$ in $\left(\alpha_{0}\right)$ of largest 


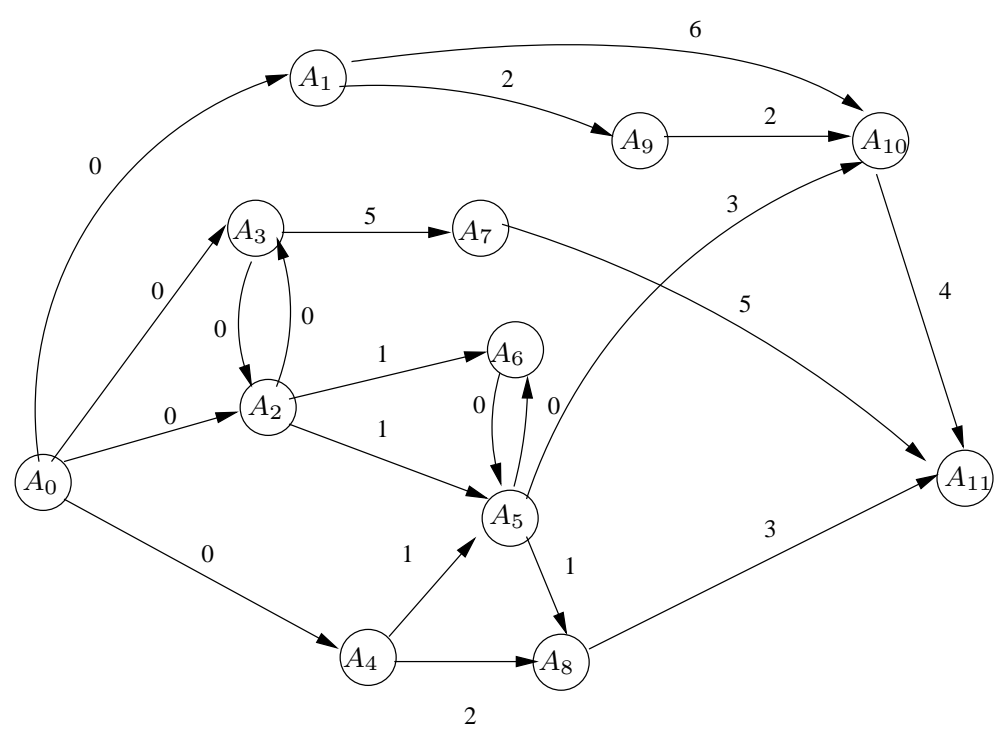

Fig. 8 Minimum time lags

$\Delta_{0, i}+p_{i}$ as possible while keeping the insertion capacity sufficient. Here we can remove activities $\{2,3,4\}$ yielding $\alpha_{t}^{*}=\{0\}$ and $\Delta_{0, n+1}\left(\alpha_{0}^{*}, \beta_{0}\right)=16$. The next dominant insertion is computed by removing $\nu\left(\alpha_{0}, \beta_{0}\right)$ from $\beta_{0}$ to obtain $\beta_{1}$ and adding $\nu^{\prime}\left(\alpha_{0}, \beta_{0}\right)$ in $\alpha_{0}$ to obtain $\alpha_{1}$. Here since $\Delta_{1,5}=-\infty$ we can insert $A_{1}$ into $\alpha_{0}$. The process is iterated for $t=1$ until there remain enough capacity in the insertion. Table 4 displays the 5 dominant insertions for the illustrative example. Insertions $\left(\alpha_{t}, \beta_{t}\right)$ are displayed as cuts on the resource flow network in Figure 9. The best insertion is obtained for $\alpha_{1}^{*}=\left\{A_{0}, A_{2}, A_{3}\right\}$ and $\beta_{1}=\left\{A_{7}, A_{8}, A_{9}, A_{10}, A_{11}\right\}$ yielding a makespan equal to 12 . Figure 11 displays the solution obtained after insertion $A_{5}$ into $\left(\alpha_{1}^{*}, \beta_{1}\right)$. 


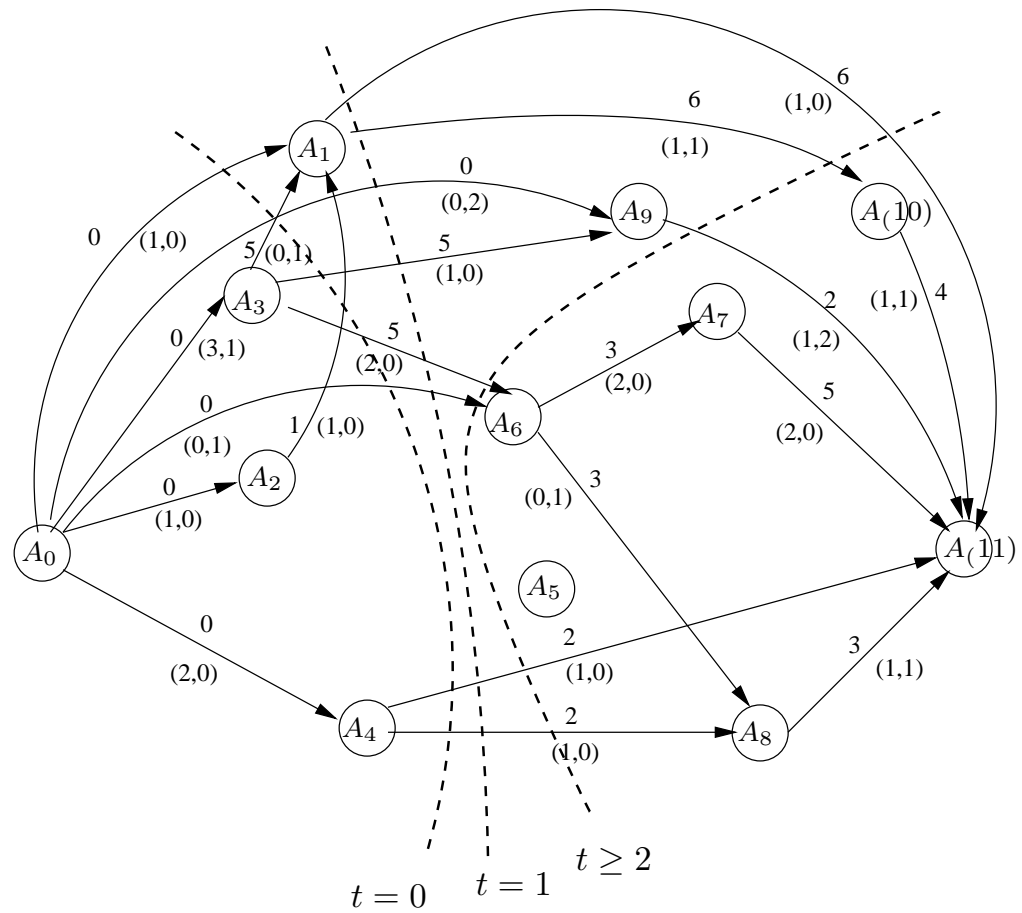

Fig. 9 Resource flow network
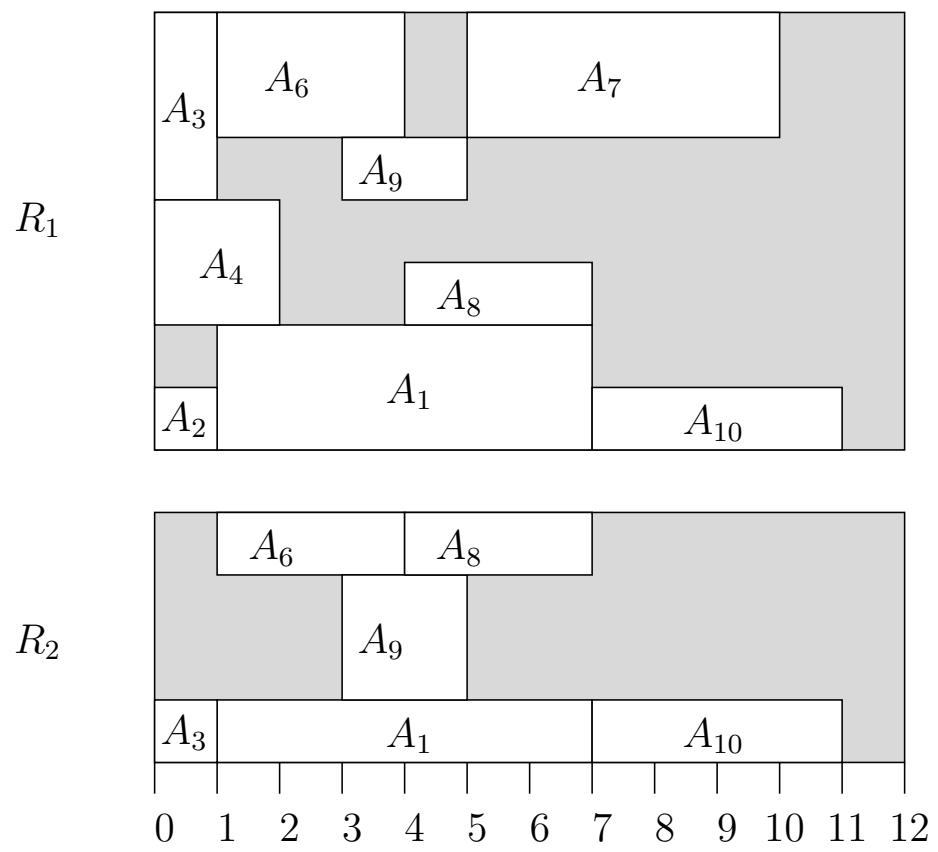

Fig. 10 Partial solution not including Activity $A_{5}$ 
Table 4 Dominant insertions

\begin{tabular}{|c|c|}
\hline$t=0$ & $\begin{array}{l}\alpha_{0}=\left\{A_{0}, A_{2}, A_{3}, A_{4}\right\} \quad \beta_{0}=\left\{A_{1}, A_{7}, A_{8}, A_{9}, A_{10}, A_{11}\right\} \\
\alpha_{0}^{*}=\left\{A_{0}\right\} \quad \Delta_{0, n+1}\left(\alpha_{0}^{*}, \beta_{0}\right)=16 \\
\nu\left(\alpha_{0}, \beta_{0}\right)=\left\{A_{1}\right\} \quad \nu^{\prime}\left(\alpha_{0}, \beta_{0}\right)=\left\{A_{1}\right\}\end{array}$ \\
\hline$t=1$ & $\begin{array}{l}\alpha_{1}=\left\{A_{0}, A_{2}, A_{3}, A_{4}, A_{1}\right\} \quad \beta_{1}=\left\{A_{7}, A_{8}, A_{9}, A_{10}, A_{11}\right\} \\
\alpha_{1}^{*}=\left\{A_{0}, A_{2}, A_{3}\right\} \quad \Delta_{0, n+1}\left(\alpha_{1}^{*}, \beta_{1}\right)=12 \\
\nu\left(\alpha_{1}, \beta_{1}\right)=\left\{A_{9}\right\} \quad \nu^{\prime}\left(\alpha_{1}, \beta_{1}\right)=\left\{A_{9}\right\}\end{array}$ \\
\hline$t=2$ & $\begin{array}{l}\alpha_{2}=\left\{A_{0}, A_{2}, A_{3}, A_{4}, A_{1}, A_{9}\right\} \quad \beta_{2}=\left\{A_{7}, A_{8}, A_{10}, A_{11}\right\} \\
\alpha_{2}^{*}=\left\{A_{0}, A_{2}, A_{3}, A_{4}, A_{9}\right\} \quad \Delta_{0, n+1}\left(\alpha_{2}^{*}, \beta_{2}\right)=15 \\
\nu\left(\alpha_{2}, \beta_{2}\right)=\left\{A_{7}\right\} \quad \nu^{\prime}\left(\alpha_{2}, \beta_{2}\right)=\emptyset\end{array}$ \\
\hline$t=3$ & $\begin{array}{l}\alpha_{3}=\left\{A_{0}, A_{2}, A_{3}, A_{4}, A_{1}, A_{9}\right\} \quad \beta_{3}=\left\{A_{8}, A_{10}, A_{11}\right\} \\
\alpha_{3}^{*}=\left\{A_{0}, A_{2}, A_{3}, A_{4}, A_{9}\right\} \quad \Delta_{0, n+1}\left(\alpha_{3}^{*}, \beta_{3}\right)=14 \\
\nu\left(\alpha_{3}, \beta_{3}\right)=\left\{A_{10}\right\} \quad \nu^{\prime}\left(\alpha_{3}, \beta_{3}\right)=\emptyset\end{array}$ \\
\hline$t=4$ & $\begin{array}{l}\alpha_{4}=\left\{A_{0}, A_{2}, A_{3}, A_{4}, A_{1}, A_{9}\right\} \quad \beta_{4}=\left\{A_{8}, A_{11}\right\} \\
\alpha_{4}^{*}=\left\{A_{0}, A_{2}, A_{3}, A_{4}, A_{9}\right\} \quad \Delta_{0, n+1}\left(\alpha_{4}^{*}, \beta_{4}\right)=13 \\
\nu\left(\alpha_{4}, \beta_{4}\right)=\left\{A_{8}\right\} \quad \nu^{\prime}\left(\alpha_{4}, \beta_{4}\right)=\emptyset\end{array}$ \\
\hline$t=5$ & $\begin{array}{l}\alpha_{5}=\left\{A_{0}, A_{2}, A_{3}, A_{4}, A_{1}, A_{9}\right\} \quad \beta_{5}=\left\{A_{11}\right\} \\
\alpha_{5}^{*}=\left\{A_{0}, A_{2}, A_{3}, A_{4}, A_{9}\right\} \quad \Delta_{0, n+1}\left(\alpha_{5}^{*}, \beta_{5}\right)=13 \\
\nu\left(\alpha_{5}, \beta_{5}\right)=\left\{A_{11}\right\} \quad \nu^{\prime}\left(\alpha_{5}, \beta_{5}\right)=\emptyset\end{array}$ \\
\hline
\end{tabular}
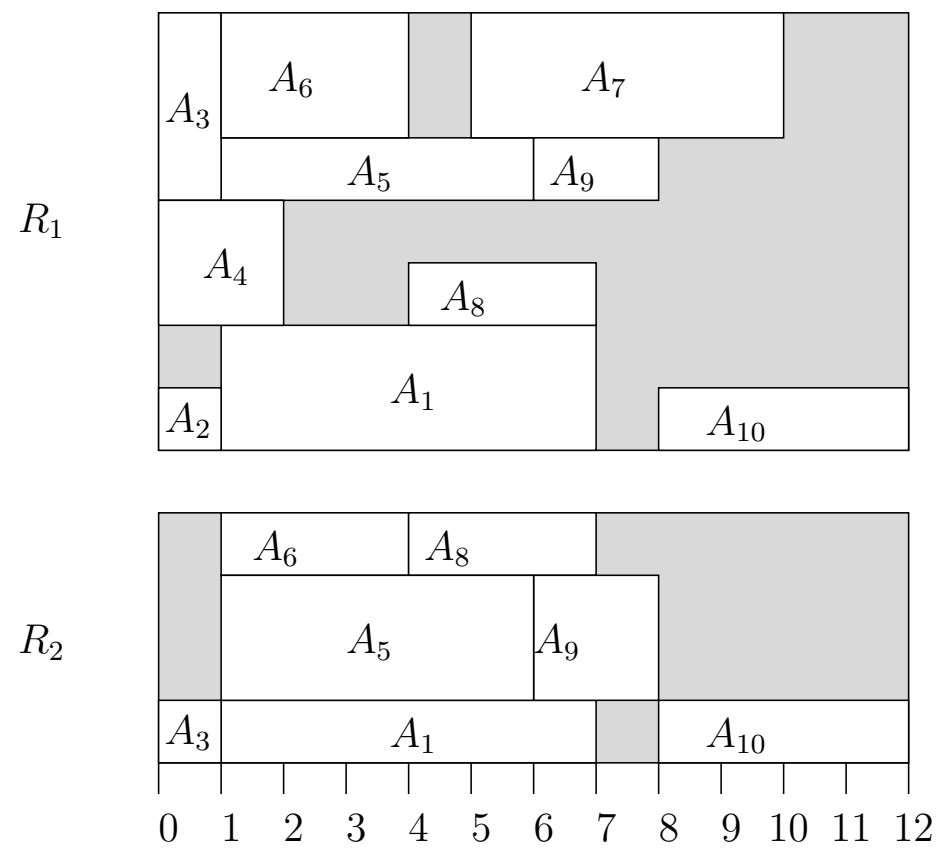

Fig. 11 Complete solution after $A_{5}$ insertion 


\section{Conclusion}

Whereas the insertion problem is polynomially solvable for the standard RCPSP (where only precedence constraints are taken into account), we showed that the introduction of minimum and maximum time lags makes the problem NP-hard. Nevertheless, when only minimum time lags are considered and when activity durations are strictly positive, the problem turns back polynomially solvable and we proposed an algorithm to solve it.

Further research may consist in designing efficient heuristics and/or branch and bound methods to solve the RCAIP with minimum and maximum time lags. A possible heuristic is to use the proposed polynomial algorithm as a basic search framework, while considering maximal time lag violation as a criterion to derive more insertion solutions. Another way of tackling insertion problems in presence of maximum time lags, would be to define another structure of the partial schedule to obtain a polyno-

mially solvable insertion problem. This remains a critical issue for designing efficient local search methods for the RCPSP/max based on activity reinsertions.

\section{References}

1. Artigues C., Michelon P. and Reusser S. Insertion techniques for static and dynamic resource-constrained project scheduling. European Journal of Operational Research 2003; 149 (2):249-267.

2. Artigues C., Roubellat F. A polynomial activity insertion algorithm in a multi-resource schedule with cumulative constraints and multiple modes. European Journal of Operational Research 2000; 127 (2):297-316.

3. Bartusch M., Möhring R.H. and Radermacher F.J. Scheduling project networks with resource constraints and time windows. Annals of Operations Research 1988; 16:201-240.

4. Brucker P. and Neyer J. Tabu-search for the multi-mode job-shop problem. OR Spektrum 1998; 20:21-28.

5. Duron C., Proth, J.M., Wardi, Y. Insertion of a random task in a schedule: a real-time approach, European Journal of Operational Research 2005, 164 (1):52-63.

6. Fortemps Ph. and Hapke M. On the Disjunctive Graph for Project Scheduling. Foundations of Computing and Decision Sciences 1997; 22:195-209.

7. Garey M. R. and Johnson D.S. Computers and intractability. A guide to the theory of NP-completeness. Freeman, 1979.

8. Gröflin H. and Klinkert A. Feasible insertions in job shop scheduling, short cycles and stable sets. European Journal of Operational Research 2007; 177 (2):763-785.

9. Kis T. and Hertz, A. A lower bound for the job insertion problem. Discrete Applied Mathematics 2003; 128 (2-3):395419.

10. Klinkert, A., Gröflin, H. and Pham-Dinh, N. Feasible Job Insertions in the Multi-ProcessorTask Job Shop. European Journal of Operational Research 2008, 185 (3): 1308-1318.

11. Leus R. and Herroelen W. Stability and resource allocation in project planning. IIE Transactions $2004 ; 36(7): 1-16$.

12. Neumann K., Schwindt C. and Zimmermann J. Project scheduling with time windows and scarce resources. Springer, 2003.

13. Vaessens R.J.M. Generalized job shop scheduling: complexity and local search. Ph.D. thesis, Eindhoven University of Technology, Rotterdam, 1995.

14. Vieira G.E., Herrmann J.W., Lin E. Rescheduling manufacturing systems: A framework of strategies, policies, and methods. Journal of Scheduling 2003, 6 (1): 39-62.

15. Vonder, S., Demeulemeester, E., Herroelen, W. A classification of predictive-reactive project scheduling procedures. Journal of Scheduling 2007, 10 (3): 195-207. 


\section{Summary of abbreviations and notations}

\begin{tabular}{|c|c|}
\hline RCPSP & resource-constrained project scheduling problem \\
\hline $\mathrm{RCPSP} / \mathrm{Max}$ & RCPSP with minimum and maximum time lags \\
\hline RCAIP & resource-constrained activity insertion problem \\
\hline$n$ & number of activities \\
\hline$G(V, E, l)$ & activity-on-node graph \\
\hline$V=\left\{A_{0}, \ldots, A_{n+1}\right\}$ & set of activities \\
\hline$E$ & set of precedence relations $\left(A_{i}, A_{j}\right)$ \\
\hline$p_{i}$ & duration of activity $A_{i}$ \\
\hline$l_{i j}$ & time lag for $\left(A_{i}, A_{j}\right) \in E$ \\
\hline$m$ & number of resources \\
\hline $\mathcal{R}=\left\{R_{1}, \ldots, R_{m}\right\}$ & set of resources \\
\hline$B_{k}$ & number of available units for resource $R_{k}$ \\
\hline$b_{i, k}$ & number of units of $R_{k}$ required by $A_{i}$ \\
\hline$S_{i}$ & start time of $A_{i}$ \\
\hline $\mathcal{A}_{t}$ & set of activities in process at time $t$ \\
\hline$(P)$ & short notation for the RCPSP/max problem \\
\hline$(\mathrm{P}-x)$ & short notation for the RCPSP $/ \max$ where $b_{x k}=0, \forall R_{k} \in \mathcal{R}$ \\
\hline$\left(\mathrm{P}_{x}\right)$ & short notation for the RCAIP where $A_{x}$ has to be inserted \\
\hline$\delta_{i, j}$ & longest path from $A_{i}$ to $A_{j}$ in $G(V, E, l)$ \\
\hline $\begin{array}{l}f_{i, j, k} \\
\mathcal{G}(f) \text { or } \mathcal{G}\end{array}$ & number of resource $R_{k}$ units transferred from $A_{i}$ to $A_{j}$ \\
\hline $\mathcal{G}(f)$ or $\mathcal{G}$ & graph induced by flow $f$ \\
\hline$F(f)$ or $F$ & set of arcs (precedence constraints) induced by flow $f$ \\
\hline$L_{i j}(f)$ or $L_{i j}$ & weight of $\operatorname{arc}\left(A_{i}, A_{j}\right)$ induced by flow $f$ \\
\hline$\Delta_{i, j}(f)$ or $\Delta_{i, j}$ & longest path from $A_{i}$ to $A_{j}$ in $\mathcal{G}(f)$ \\
\hline$q_{i, j, k}$ & part of flow $f_{i, j, k}$ rerouted to the inserted activity \\
\hline$(\alpha, \beta)$ & ordered pair of set of activities representing an insertion \\
\hline$\alpha$ & set of possible resource predecessors \\
\hline$\beta$ & set of possible resource successors \\
\hline$Q_{k}(\alpha, \beta)$ & amount of $R_{k}$ units available for insertion in $(\alpha, \beta)$ \\
\hline $\mathcal{G}(\alpha, \beta)$ & graph issued from the insertion of $A_{x}$ in $(\alpha, \beta)$ \\
\hline$F(\alpha, \beta)$ & set of arcs issued from the insertion of $A_{x}$ in $(\alpha, \beta)$ \\
\hline$L_{i, j}(\alpha, \beta)$ & weight of $\operatorname{arc}\left(A_{i}, A_{j}\right)$ after insertion of $A_{x}$ in $(\alpha, \beta)$ \\
\hline$\Delta_{i, j}(\alpha, \beta)$ & longest path from $A_{i}$ to $A_{j}$ in $\mathcal{G}(\alpha, \beta)$ \\
\hline $\mathcal{C}_{q}(\alpha, \beta)$ & set of type $q$ cycles in $\mathcal{G}(\alpha, \beta)(q=1,2,3)$ \\
\hline $\mathcal{L}_{q}(\alpha, \beta)$ & length of the longest cycle in $\mathcal{G}(\alpha, \beta)(q=1,2,3)$ \\
\hline $\mathcal{M}_{q}(\alpha, \beta)$ & length of the longest path of type $q$ in $\mathcal{G}(\alpha, \beta)(q=1,2,3)$ \\
\hline$\gamma$ & set of non-dummy activities linked with $A_{x}$ by a synchronization constraint \\
\hline$\mu(\alpha)$ & set of activities $i \in \alpha$ of largest $\Delta_{0, i}+p_{i}$ \\
\hline$\nu(\alpha)$ & set of activities $i \in \beta$ of largest $\Delta_{i, n+1}$ \\
\hline$\nu^{\prime}(\alpha)$ & subset of activities $i \in \nu$ such that $\Delta_{x, i}=-\infty$ \\
\hline
\end{tabular}

